Supporting Information for:

\title{
Conformation, and Charge Tunneling Through Molecules in SAMs
}

Lee Belding, ${ }^{\mathrm{a} \dagger}$ Samuel E. Root, ${ }^{\mathrm{a} \dagger}$ Yuan Li, ${ }^{\mathrm{a}}$ Junwoo Park, ${ }^{\mathrm{a}}$ Mostafa Baghbanzadeh, ${ }^{\mathrm{a}}$ Edwin Rojas, ${ }^{a}$ Priscilla F. Pieters, ${ }^{\mathrm{a}}$ Hyo Jae Yoon, ${ }^{\mathrm{b}}$ and George M. Whitesides ${ }^{{ }^{*}}$

a Department of Chemistry and Chemical Biology, Harvard University

12 Oxford Street, MA 02138

${ }^{b}$ Department of Chemistry, Korea University, Seoul, 02841, Korea

* Author to whom correspondence should be addressed.

$\uparrow$ These authors contributed equally.

S1. Materials.

S2. Junction measurements using "selected" conical tips of EGaIn.

S3. EGaIn measurement protocol.

S4. Angular-dependent X-ray photoelectron spectroscopy (XPS).

S5. Computational methods

S6. Comparison between experimental and simulated monolayer thickness

S7. Summary of XPS data

S8. Summary of tunneling Data

S10. Summary of Simulation Data

S11. Synthesis

S12. Contact angle data 


\section{S1. Materials.}

All organic solvents were analytical grade (99\%, Sigma-Aldrich) and were used as supplied unless otherwise specified. Starting materials were commercially available $(\geq 98 \%$, Sigma-Aldrich). The synthesis of the branched and unbranched compounds is described below. All disulfide-based compounds were stored under a $\mathrm{N}_{2}$ atmosphere and at $<4^{\circ} \mathrm{C}$ to avoid oxidation to the corresponding sulfonate or sulfonic acid. To ensure that the compounds were free of contaminants, all stored compounds were checked by ${ }^{1} \mathrm{H}$ NMR prior to use; any impurities were removed by silica gel column chromatography, using a solvent system specified below in their synthesis.

\section{S2. Junction measurements using "selected" conical tips of EGaIn.}

We use EGaIn (eutectic Ga-In; 74.5\% Ga, 25.5\% In) as a non-damaging top electrode for measuring currents in junctions having the structure $\mathrm{Ag}^{\mathrm{TS}} / \mathrm{SAM} / / \mathrm{Ga}_{2} \mathrm{O}_{3} / \mathrm{EGaIn}^{1-2}$ This liquid metal forms a self-passivating oxide layer of $\mathrm{Ga}_{2} \mathrm{O}_{3}(0.7 \mathrm{~nm}$ thick on average) when exposed to air. The mechanical properties of the electrically conducting $\mathrm{Ga}_{2} \mathrm{O}_{3}$ film ${ }^{3}$ that forms on its surface facilitates the formation of sharp conical tips, facilitating the formation of a small geometrical contact area of $\sim 25 \mu \mathrm{m}$ in diameter, or $\sim 490 \mu \mathrm{m}^{2}$ in geometrical contact area estimated by microscopy. We selected EGaIn conical tips that were free of visible surface asperities; conical tips that had visible irregularities (by optical microscopy) were not used. ${ }^{4}$

\section{S3. EGaIn measurement protocol.}

The SAMs were prepared on template stripped substrates ${ }^{5}$ using disulfides as the anchoring group according to protocols previously reported, ${ }^{6-7}$ with an incubation period of 18 hours (except for $\mathbf{1}_{(4, \mathrm{H})}$ and $\mathbf{6}_{(4,4)}$, which showed low surface coverage- 0.4 relative to 
decanethiol—after 18 hours, and were thus incubated for 32 hours). For each compound investigated, we measured 7 junctions (individual points of contact between $\mathrm{Ga}_{2} \mathrm{O}_{3} / \mathrm{EGaIn}$ tip and the SAM) on three independent substrates (for a total of 21 junctions). Each junction is comprised of $21 J(\mathrm{~V})$ traces $(\mathrm{A} J(\mathrm{~V})$ trace involved sweeping from $0 \mathrm{~V} \rightarrow+1.0 \mathrm{~V} \rightarrow-1.0 \mathrm{~V} \rightarrow$ $0 \mathrm{~V}$ in steps of $50 \mathrm{mV}$, with a delay of $0.2 \mathrm{~s}$ between each step in applied bias, while measuring $J$ at each bias). Each $J(\mathrm{~V})$ trace yielded two values of $J$ for each value of applied bias. Thus, we collected $>800$ values (N) of $J$ at every applied bias for each molecule.

\section{S4. Angular-dependent X-ray photoelectron spectroscopy (XPS).}

Angle-dependent XPS measurements were used to calculate the thickness of the SAMs, by measuring the positions of sulfa (S) atoms with respect to vacuum (i.e., measuring how "buried" these atoms are in the SAMs). The position of the analyzer was fixed at a $50^{\circ}$ angle from the incident $\mathrm{X}$-ray. The emission angle $(\theta)$ was defined as the angle between the axis of the analyzer and the substrate surface. The incident angle $(\gamma)$ was defined as the angle between X-ray incidence and the substrate surface. The $\mathrm{S} 2 p$ spectra were collected at 5 angles $\left(\theta=90^{\circ}, 75^{\circ}\right.$, $60^{\circ}, 45^{\circ}, 30^{\circ}$ ), by rotating the sample holder. To reduce the difference in detected area, induced by sample rotation, the effective intensity $\left(I_{\theta}\right)$ is given by

$$
I_{\theta}=I \cos \left(90^{\circ}-\gamma\right)
$$

where $I$ is the integration of the S $2 p$ peak. We determined the monolayer thickness $d_{\mathrm{SAM}}$ (in nm) with the sum of theoretical distance of the Ag-S bond $(\sim 1.8 \AA)$ and $d$ (defined as the thickness of the overlayer from the sulfur to the vacuum), as shown in eq. 2. $d$ is estimated using eq. 3 which has been reported before. ${ }^{8-9}$

$$
\begin{gathered}
d_{\mathrm{SAM}}=d+d_{\mathrm{Ag}-\mathrm{S}} \\
I_{\theta}=I_{0} \exp (-d /(\lambda \sin \theta))
\end{gathered}
$$


where $\lambda$ is the inelastic mean free path $(\lambda=25 \AA)$ when the kinetic energy of the photoelectrons is at $\sim 1280 \mathrm{eV}, I_{0}$ is the initial intensity from the sulfur atom. In our experiments, we used the intensity of $\mathrm{S}$ to calculate the value of $d$ by fitting eq. 4 (derived from eq. 3) to a plot of the $\ln$ $\left(I_{\theta, \mathrm{S}}\right)$ vs. $1 / \sin \theta$, and the slope is $d / \lambda$ (Figure S1). Finally, we calculated the values of $d_{\mathrm{SAM}}$ shown in the Table S1-S5.

$$
\ln \left(I_{\theta, \mathrm{S}}\right)=\ln \left(I_{0, \mathrm{~S}}\right)-d / \lambda \sin \theta
$$

Instead of determining the absolute surface coverages $\left(\Gamma_{\mathrm{SAM}}\right)$, we determined the relative values of $\Gamma_{\mathrm{SAM}}$. The intensity of the $\mathrm{Au}\left(I_{\mathrm{Au}}\right)$ signal is attenuated by the SAM depending on $d$ and the number of molecules absorbed on Au surfaces, the surface coverage. Since we know $d$, and the surface coverage of a simple Au-SC 10 SAM is known, we can obtain the relative $\Gamma_{\mathrm{SAM}}$ values by comparing $I_{\mathrm{Au}}$ for the different types of SAMs given by eq. 5 .

$$
I_{0, \mathrm{Au}-\mathrm{S}}=I_{\mathrm{Au}} / \exp \left(-d / \lambda_{\mathrm{Au}}\right)
$$

Here, $I_{0, \text { Au-S }}$ is the Au signal attenuated by the sulphur atoms. Since the nature of the Au-S bond is the same for all the SAMs (in terms of bond angle and length), the difference between the $I_{0}$, Au-S of two different SAMs can be solely related to $\Gamma_{\mathrm{SAM}}$. The theoretical value of $\Gamma_{\mathrm{SAM}}$ of $\mathrm{SC}_{10}$ SAMs on $\mathrm{Au}(111)$ surface has been reported before and is $1.0 \times 10^{-9} \mathrm{~mol} / \mathrm{cm}^{2} .{ }^{10}$ Thus, we compared the values of $I_{0, \mathrm{Au}-\mathrm{S}}$ of the SAMs against the $I_{0, \mathrm{Au}-\mathrm{S}}$ of $\mathrm{SC}_{10} \mathrm{SAMs}$ to estimate the relative $\Gamma_{\mathrm{SAM}}$ values of the SAMs in this study. 

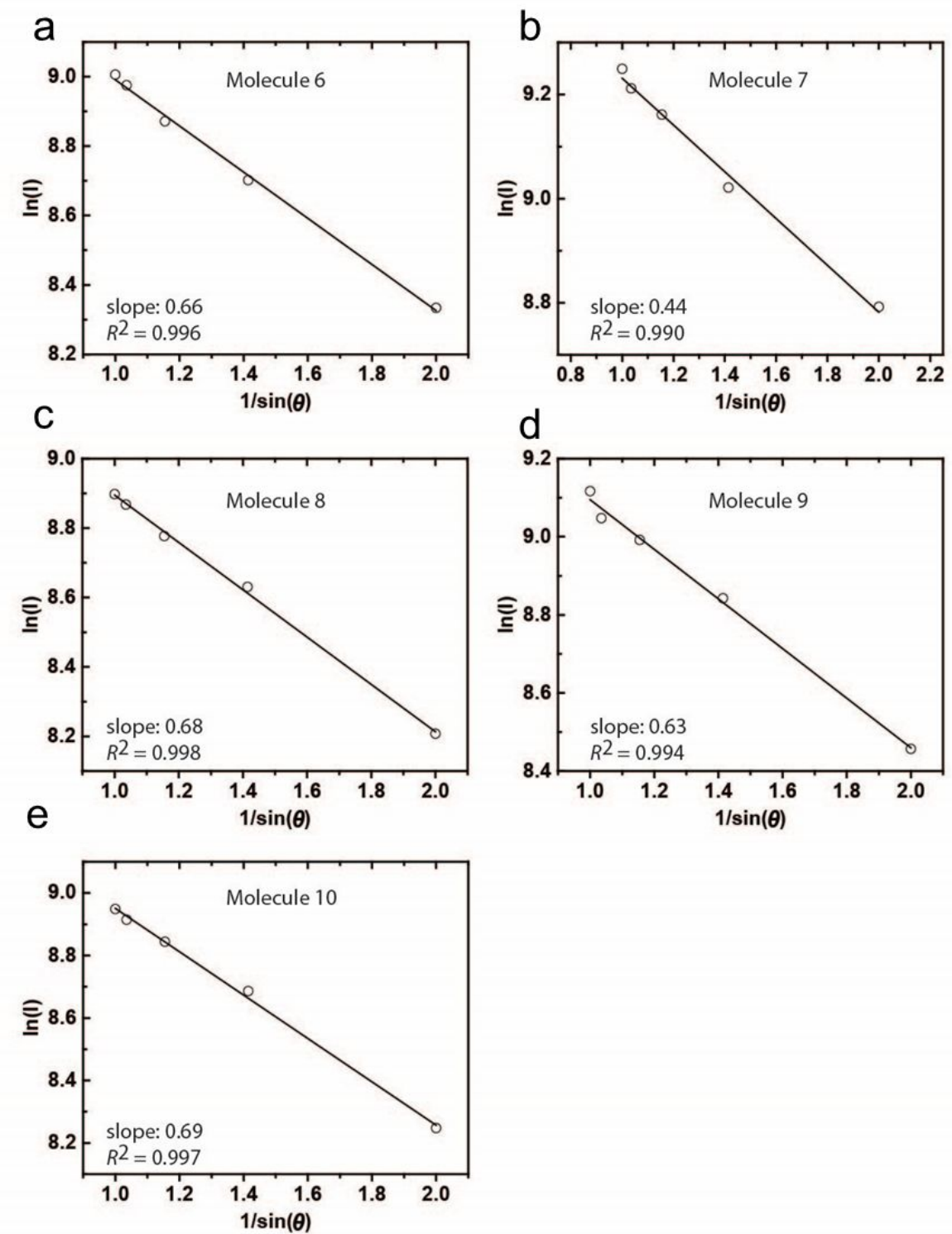

Figure S1. Determination of monolayer thickness from ARXPS measurements. Plots of $\ln \left(I_{\theta, \mathrm{S}}\right)$ vs. $1 / \sin \theta$ showing the quality of the linear fit $\left(\mathrm{R}^{2}>0.99\right)$ for the symmetric tertiary amide series, (a) $\mathbf{6}_{(4,4)}$, (b) $\mathbf{7}_{(6,6)}$, (c) $\mathbf{8}_{(8,8)}$, (d) $\mathbf{9}_{(10,10)}$, (e) $\mathbf{1 0}_{(12,12)}$. 
The ARXPS measurement of S $2 p$ spectra of one SAM required about 20 mins to complete. Because this long time X-ray irradiation may potentially damage the SAMs we measured, we recorded the C1s spectra before and after acquisition of S 2p spectra. Figure S2 shows that we did not find any observable irradiation damage to the SAM we measured.

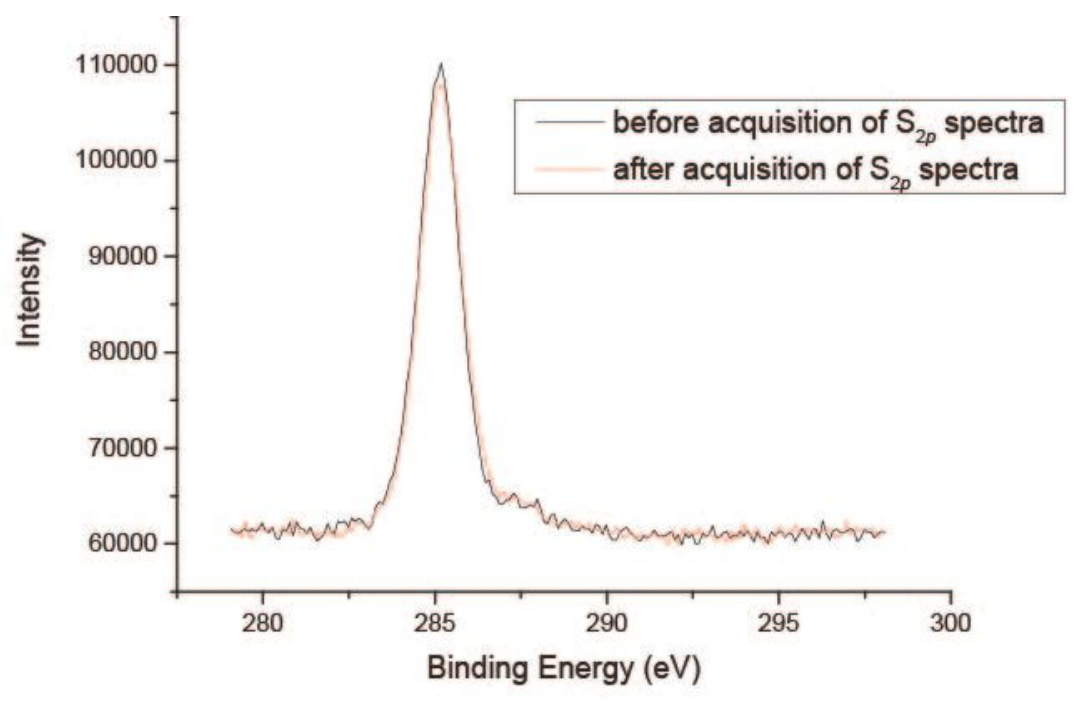

Figure S2. A plot showing the C1s spectra taken before and after acquisition of S $2 p$ spectra, demonstrating that an X-ray irradiation of $\sim 20$ minutes did not damage the samples. 

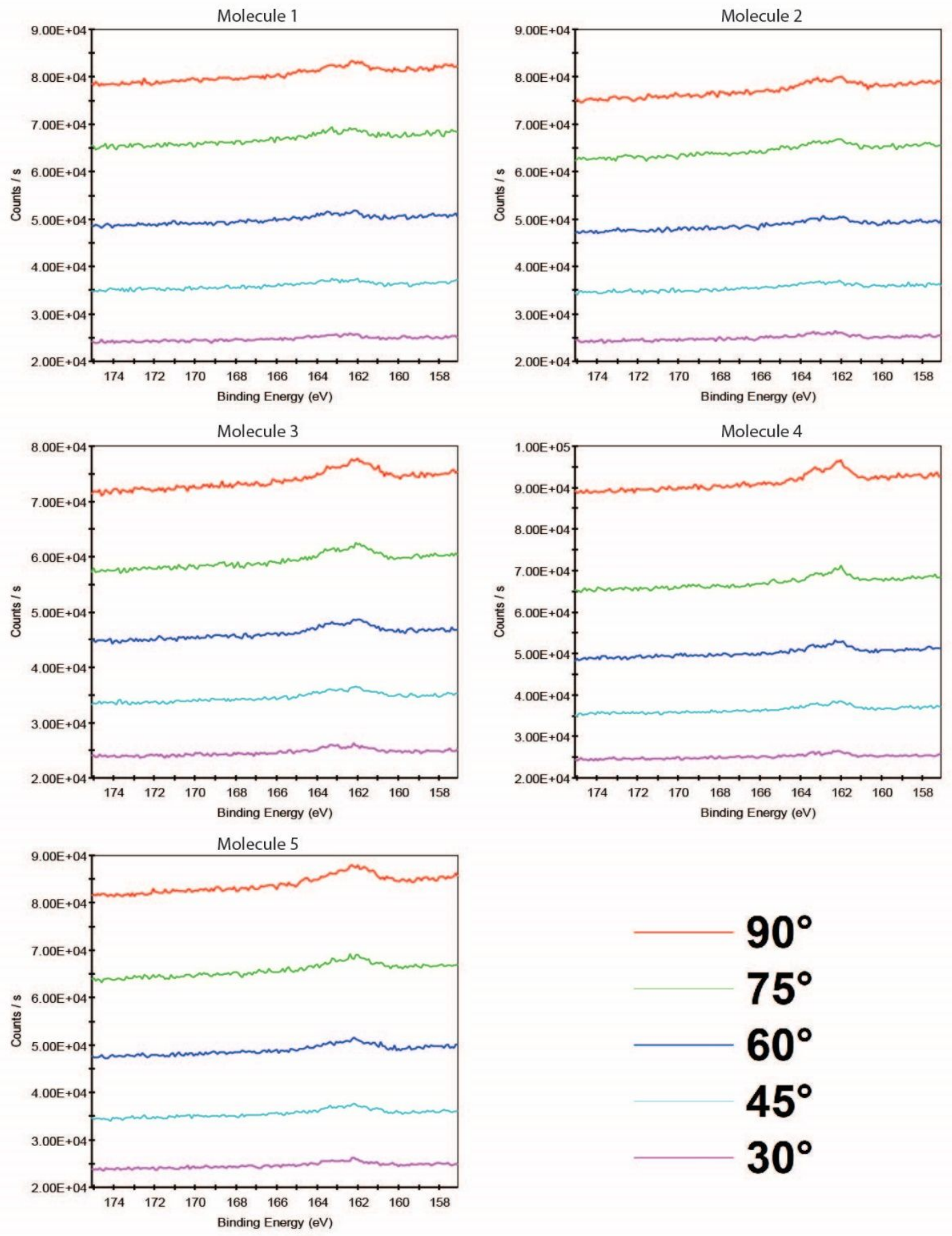

\section{$90^{\circ}$}

$75^{\circ}$

$60^{\circ}$

$45^{\circ}$

$30^{\circ}$

Figure S3. The S2p spectra of molecule 1-5 taken at 5 different take-off angles. 


\section{S5. Computational methods}

All MD simulations were run on 12 processors using LAMMPS and visualized using OVITO. ${ }^{1,2}$ The initial conditions for the MD simulations were prepared by arranging 256 equally spaced SAM molecules, at a specific area of $22.6 \AA^{2} /$ chain, on a gold (111) slab of dimensions $81.6 \times 70.7 \times 6.7 \AA^{3}$. These dimensions were chosen following existing works in the literature ${ }^{11}$ and due to constraints on computational resources with the large number of different simulations required for all of the molecules considered in this study. The simulation box was specified with periodic boundaries in the $\mathrm{X}, \mathrm{Y}$ dimensions, and fixed boundaries in the $\mathrm{Z}$ dimension. Dynamics were run using the generic OPLS-2005 forcefield that includes Lennard-Jones and electrostatic pairwise interactions; harmonic bond and angle potentials; and dihedral potentials described by Fourier series. ${ }^{3}$ Lennard-Jones interactions were truncated and shifted to zero at a cutoff radius of $12 \AA$. Long-range electrostatic interactions in the $\mathrm{X}, \mathrm{Y}$ dimensions were treated using an Ewald summation to a specified force accuracy of $1 \times 10^{-4} \mathrm{kcal} / \mathrm{mol} \cdot \AA$. Due to the quasi-2D nature of the system, long-range electrostatic forces $(\sim 10 \mathrm{~nm})$ in the $\mathrm{Z}$ dimension were set to zero. Specific parameters for the gold-sulfur interaction were taken from the work of Ghorai and Glotzer. ${ }^{4}$ The gold-sulfur bond was treated with a pairwise additive Morse potential. For the linear amide series, the pairwise Lennard-Jones interaction between the amide hydrogen atom and the oxygen atom was modified to $\sigma=1.5 \AA$, and $\epsilon=0.30 \mathrm{kcal} / \mathrm{mol}$, following a recently developed force field that has been shown to give an accurate description of hydrogen bonding in proteins. $^{5}$

Once the initial conditions were specified, we ran a conjugate gradient energy minimization. Subsequently, the system was thermalized using a Nose-Hoover thermostat (time constant $=100 \mathrm{fs}$ ) that increased the temperature from 0 to $400 \mathrm{~K}$ over the course of $5 \mathrm{~ns}$, using a 
timestep of 1 fs. Dynamics were then run at $400 \mathrm{~K}$ for $10 \mathrm{~ns}$ and cooled down to $300 \mathrm{~K}$ over the course of $5 \mathrm{~ns}$. All results were sampled from subsequent dynamics run at $300 \mathrm{~K}$ and temporally averaged over $5 \mathrm{~ns}$. The thickness of the monolayer was calculated as the vertical difference between sulfur and the terminal hydrogen atom (i.e. with the maximum z-position) in each molecule, averaged over all the molecules and over time. The C-C-C-C and C-N-C-C dihedral angles were directly outputted from LAMMPS and were binned with $1^{\circ}$ intervals and normalized over all the angles in each simulation to obtain probability distributions.

For many of the tertiary SAMs, we observed that the initially high surface coverage $(22.6$ $\AA^{2} /$ chain) led to large forces that caused molecules to desorb from the surface during the thermal equilibration procedure. This observation was consistent with our experimental measurements, which showed that the tertiary amides formed SAMs with a lower molar packing density. All molecules that desorbed from the surface were subsequently deleted from the simulation using a cutoff determined by inspection (Figure S4). Similar trends were observed between predicted surface coverage and experimentally measured surface coverage; however, the predicted coverage was generally slightly less than the experimental values. 


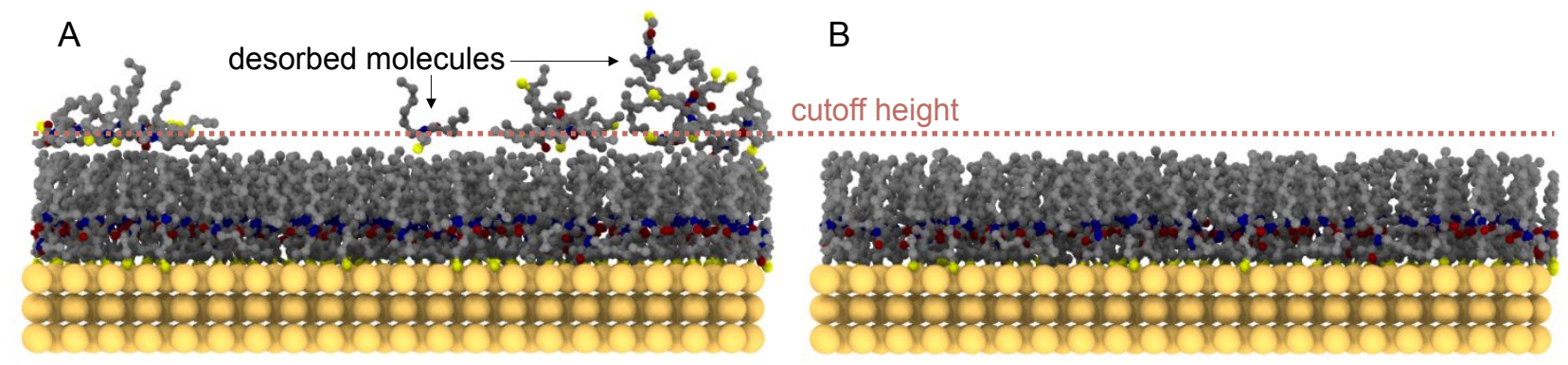

Figure S4. Methodology for deleting desorbed molecules. Molecules were initially deposited on the surface at a packing density of $22.6 \AA^{2} /$ chain, irrespective of molecular structure. (A) Following energy minimization and thermal equilibration of the system, molecules were observed to spontaneously desorb from the surface to an extent that was dependent upon the molecular structure. (B) A cutoff height was determined by visual inspection, and desorbed molecules, containing one or more atoms above this cutoff height, were deleted from the simulation. 
The fraction of branches pointing down, $f_{\text {down }}$, was calculated from a normalized histogram of the positions of all of the terminal methyl groups over a duration of $5 \mathrm{~ns}$, to obtain an ensemble-averaged probability distribution function (PDF). The PDF contained 1-3 peaks depending upon the molecular structure (Figure S5A-C). That is, linear molecules had a single peak (indicating all were pointing up), symmetrically branched molecules had two distinct peaks and asymmetrically branched molecules had 3 distinct peaks. This PDF was subsequently integrated to calculate the cumulative distribution function (CDF) (Figure S5D-F). For each series a threshold z-value, shown by the vertical dashed line in Figure S5D-F, was chosen by manual inspection and the value of the CDF at that z-position was taken as the fraction of branches pointing down. For asymmetrically branched molecules, since the longer branch always

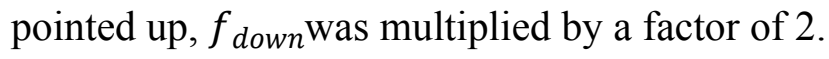



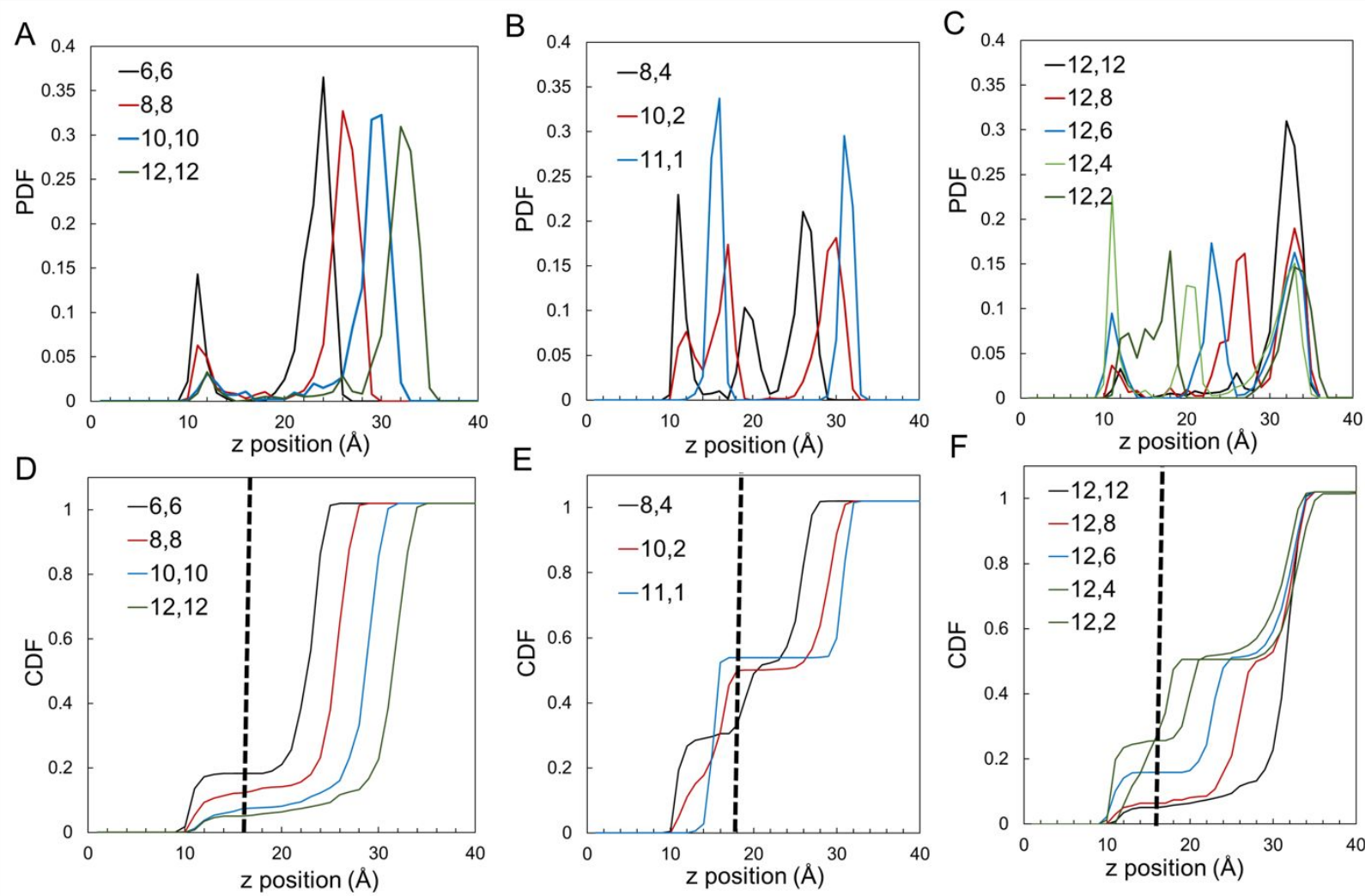

Figure S5. Statistics of the orientation of branched chains. Probability distribution functions for the z-position of the terminal methyl carbons for (A) the symmetrically branched series, (B) the asymmetrically branched series 1 , and (C) the asymmetrically branched series 2 . Cumulative distribution functions for the z-position of the terminal methyl carbons for (D) the symmetrically branched series, (E) the asymmetrically branched series 1, and (F) the asymmetrically branched series 2 . The dashed vertical line indicates the threshold z-value above or below which branches were considered to be pointing up or down. 


\section{S6. Comparison between experimental and simulated monolayer thickness}

In this work we obtained values for the thickness of the monolayers by two methods: angle-resolved x-ray photoelectron spectroscopy (ARXPS) and molecular dynamics (MD) simulation. The thickness, as determined by ARXPS, relies on the attenuation of the signal arising from the sulfur $2 p$ photoelectron as the angle between the sample and the incident X-ray decreases and it must travel further through the monolayer. The simulated thickness of the monolayer was calculated as the vertical difference between the sulfur atom and the hydrogen atom in each molecule with the maximum z-position, averaged over all the molecules and over time.

The simulated thicknesses may differ from the actual monolayer thicknesses for several reasons: (i) the angle made between the molecules and the Au surface may not be accurately captured by the simulation, (ii) the packing density may not be accurately captured by the simulation, and (iii) the preferred structure of the SAM may not be accurately captured by the simulation. We also highlight the uncertainty in the experimentally derived calculations of thickness (particularly for the branched and disordered SAMs), due to undefined influence of differences in packing density on the intensity of the S $2 p$ signal. Despite these possible differences, we observed very similar trends between the experimentally determined and computationally derived values of thickness, which are summarized in table S15. For example, the slope of the line made from a graph of thickness vs. the number of atoms in the longest chain was the same (to within one decimal place in units of $\AA$ /atom) for three of the four series (the exception being symmetrically branched amides, which differed by $0.2 \AA$ /atom). The thicknesses obtained from the MD simulations were consistently larger (by $\sim 0.5-3 \AA$ ) than those obtained using XPS. The difference in thickness obtained with MD and XPS was the smallest for linear 
amides (on average $0.51 \AA$ ) and the largest for Disordered Series I (3.0 $\AA$ ). For comparison, the average C-C bond length is $1.56 \AA$.

The similarity in the trends between experimentally (XPS) and computationally (MD) derived values of thickness, suggests that both methods are reliable. Moreover, by comparing relative trends between sets of similar compounds with small, incremental changes in structure, we limit the effects of differences in absolute values of thickness. In our discussions involving thickness, we refer to the experimentally determined thickness (i.e. the values obtained empirically by XPS), unless otherwise specified.

Table S1. Differences in the values of the thickness $(h)$ of SAMs obtained from XPS measurements and MD simulations.

Slope of the line made from a graph of thickness and the total number of atoms in the longest chain length:
Average of all the differences in thickness obtained from XPS and MD between each $\mathrm{SAM}$ in the series

\section{XPS MD}

Secondary amides

$1.1 \AA$ atom

$1.1 \AA$ atom

$0.51 \AA$

Symmetric tertiary amides

$1.0 \AA$ /atom $\quad 1.2 \AA /$ atom

$2.1 \AA$

Series I amides

$1.0 \AA$ atom $\quad 1.0 \AA /$ atom

$3.0 \AA$

Series II amides

$0.1 \AA$ /atom $\quad 0.1 \AA$ atom

$2.9 \AA$

\section{S7. Summary of XPS data}

Table S2. Summary of the relative surface coverage $(d)$ and thickness $(h)$ of SAMs made from secondary amides, calculated from ARXPS.

$\begin{array}{lcc}\text { Molecule } & \text { Relative surface coverage }\left(\times \mathbf{1 0}^{-\mathbf{9}} \mathbf{~ m o l} / \mathbf{c m}^{\mathbf{2}}\right) & \text { thickness }(\AA) \\ \text { decanethiol } & 1.0 \pm 0.12 & 12.5 \pm 1.5 \\ \mathbf{2}_{(6, \mathrm{H})} & 1.0 \pm 0.12 & 11.0 \pm 1.5 \\ \mathbf{3}_{(8, \mathrm{H})} & 0.9 \pm 0.12 & 13.9 \pm 1.5 \\ \mathbf{4}_{(10, \mathrm{H})} & 1.0 \pm 0.12 & 14.9 \pm 1.5 \\ \mathbf{5}_{(12, \mathrm{H})} & 1.0 \pm 0.12 & 17.1 \pm 1.5\end{array}$


Table S3. Summary of the relative surface coverage $(d)$ and thickness $(h)$ of SAMs made from symmetric tertiary amides, calculated from ARXPS.

$\begin{array}{lcc}\text { Molecule } & \text { Relative surface coverage }\left(\times \mathbf{1 0}^{-\mathbf{9}} \mathbf{~ m o l} / \mathbf{c m}^{\mathbf{2}}\right) & \text { thickness }(\AA) \\ \mathbf{6}_{(4,4)} & 0.8 \pm 0.12 & 9.4 \pm 1.5 \\ \mathbf{7}_{(6,6)} & 0.8 \pm 0.12 & 11.0 \pm 1.5 \\ \mathbf{8}_{(8,8)} & 0.73 \pm 0.12 & 11.4 \pm 1.5 \\ \mathbf{9}_{(10,10)} & 0.78 \pm 0.12 & 13.8 \pm 1.5 \\ \mathbf{1 0}_{(12,12)} & 0.82 \pm 0.12 & 16.7 \pm 1.5\end{array}$

Table S4. Summary of the relative surface coverage $(d)$ and thickness $(h)$ of Series I SAMs, calculated from ARXPS.

$\begin{array}{lcc}\text { Molecule } & \text { Relative surface coverage }\left(\times \mathbf{1 0}^{-\mathbf{9}} \mathbf{~ m o l} / \mathbf{c m}^{\mathbf{2}}\right) & \text { Thickness }(\AA) \\ \mathbf{6}_{(6,6)} & 0.8 \pm 0.12 & 11.0 \pm 1.5 \\ \mathbf{7}_{(8,4)} & 0.8 \pm 0.12 & 9.3 \pm 1.5 \\ \mathbf{8}_{(10,2)} & 0.8 \pm 0.12 & 13.1 \pm 1.5 \\ \mathbf{9}_{(11,1)} & 0.9 \pm 0.12 & 11.5 \pm 1.5 \\ \mathbf{5}_{(12, \mathrm{H})} & 1.0 \pm 0.12 & 17.1 \pm 1.5\end{array}$

Table S5. Summary of the relative surface coverage $(d)$ and thickness $(h)$ of SAMs made from series II amides, calculated from ARXPS.

$\begin{array}{lcc}\text { Molecule } & \text { Relative surface coverage }\left(\times \mathbf{1 0}^{-\mathbf{9}} \mathbf{~ m o l} / \mathbf{c m}^{\mathbf{2}}\right) & \text { thickness }(\AA) \\ \mathbf{1 4}_{(12,2)} & 0.86 \pm 0.12 & 14.2 \pm 1.5 \\ \mathbf{1 5}_{(12,4)} & 0.9 \pm 0.12 & 14.4 \pm 1.5 \\ \mathbf{1 6}_{(12,6)} & 0.9 \pm 0.12 & 15.2 \pm 1.5 \\ \mathbf{1 7}_{(12,8)} & 0.89 \pm 0.12 & 16.5 \pm 1.5 \\ \mathbf{1 8}_{(12,10)} & 0.88 \pm 0.12 & 16.4 \pm 1.5 \\ \mathbf{1 0}_{(12,12)} & 0.82 \pm 0.12 & 16.7 \pm 1.5\end{array}$




\section{S8. Summary of Tunneling Data}

Table S6. Measured values of $\log |J|$ at $+1.0 \mathrm{~V}$ and $-1.0 \mathrm{~V}$ and the corresponding rectification ratios $(r+)$ for secondary amides $\mathbf{1}$ - 5 .

$$
\mathrm{V}=+\mathbf{1 . 0} \mathrm{V}=\mathbf{- 1 . 0 \mathrm { V }}
$$

\begin{tabular}{ccccccc} 
Molecule & $\log |\boldsymbol{J}|$ & $\boldsymbol{\sigma}_{\mathbf{l o g}}$ & $\log |\boldsymbol{J}|$ & $\boldsymbol{\sigma}_{\log }$ & $\boldsymbol{r}^{+\boldsymbol{a}}$ & $\boldsymbol{\sigma}_{\boldsymbol{r +}}$ \\
\hline $\mathbf{1}_{(4, \mathrm{H})}$ & 1.13 & 0.23 & 0.36 & 0.27 & 6.15 & 1.28 \\
$\mathbf{2}_{(6, \mathrm{H})}$ & 0.49 & 0.22 & -0.40 & 0.30 & 7.08 & 1.36 \\
$\mathbf{3}_{(8, \mathrm{H})}$ & -0.16 & 0.42 & -1.00 & 0.44 & 7.24 & 1.33 \\
$\mathbf{4}_{(10, \mathrm{H})}$ & -0.66 & 0.27 & -1.66 & 0.46 & 11.59 & 2.61 \\
$\mathbf{5}_{(12, \mathrm{H})}$ & -1.50 & 0.35 & -2.5 & 0.43 & 10.42 & 1.40 \\
${ }^{a} r^{+}=<|J(+1 \mathrm{~V})| /|J(-1 \mathrm{~V})|>$ & & & &
\end{tabular}

Table S7. Measured values of $\log |J|$ at $+0.5 \mathrm{~V}$ and $-0.5 \mathrm{~V}$ and the corresponding rectification ratios $(r+)$ for secondary amides $\mathbf{1}$ - 5 .

\begin{tabular}{|c|c|c|c|c|c|}
\hline \multirow[b]{2}{*}{ Molecule } & \multicolumn{2}{|c|}{$\mathrm{V}=+0.5 \mathrm{~V}$} & \multicolumn{2}{|c|}{$V=-0.5 V$} & \multirow[b]{2}{*}{$r^{+a}$} \\
\hline & $\log |J|$ & $\sigma_{\log }$ & $\log |J|$ & $\sigma_{\log }$ & \\
\hline $\mathbf{1}_{(4, \mathrm{H})}$ & 0.24 & 0.27 & 0.01 & 0.29 & 1.70 \\
\hline $\mathbf{2}_{(6, \mathrm{H})}$ & -0.6 & 0.2 & -0.8 & 0.3 & 1.58 \\
\hline $\mathbf{3}_{(8, \mathrm{H})}$ & -1.16 & 0.44 & -1.49 & 0.46 & 2.14 \\
\hline $\mathbf{4}_{(10, \mathrm{H})}$ & -1.60 & 0.35 & -1.93 & 0.34 & 1.86 \\
\hline $\mathbf{5}_{(12, \mathrm{H})}$ & -2.84 & 0.30 & -3.10 & 0.38 & 1.82 \\
\hline
\end{tabular}


Table S8. Measured values of $\log |J|$ at $+1.0 \mathrm{~V}$ and $-1.0 \mathrm{~V}$ and the corresponding rectification ratios $(r+)$ for symmetric tertiary amides $\mathbf{6}-\mathbf{1 0}$.

\begin{tabular}{|c|c|c|c|c|c|c|}
\hline \multirow[b]{2}{*}{ Molecule } & \multicolumn{2}{|c|}{$\mathrm{V}=+1.0 \mathrm{~V}$} & \multicolumn{2}{|c|}{$\mathrm{V}=-\mathbf{1 . 0} \mathrm{V}$} & \multirow[b]{2}{*}{$r^{+a}$} & \multirow[b]{2}{*}{$\sigma_{r+}$} \\
\hline & $\log |J|$ & $\sigma_{\log }$ & $\log |J|$ & $\sigma_{\log }$ & & \\
\hline $\mathbf{6}_{(4,4)}$ & 0.33 & 0.13 & -0.4 & 0.15 & 5.6 & 1.25 \\
\hline $7_{(6,6)}$ & -0.1 & 0.4 & -0.88 & 0.43 & 6.13 & 1.78 \\
\hline $\mathbf{8}_{(8,8)}$ & -0.17 & 0.47 & -1.24 & 0.53 & 9.89 & 1.71 \\
\hline $\mathbf{9}_{(10,10)}$ & -0.71 & 0.46 & -1.74 & 0.44 & 12.39 & 1.96 \\
\hline $\mathbf{1 0}_{(12,12)}$ & -1.86 & -0.1 & -2.86 & 0.08 & 11.22 & 2.27 \\
\hline
\end{tabular}

Table S9. Measured values of $\log |J|$ at $+0.5 \mathrm{~V}$ and $-0.5 \mathrm{~V}$ and the corresponding rectification ratios $(r+)$ for symmetric tertiary amides $6-10$.

\begin{tabular}{cccccc} 
& \multicolumn{6}{c}{$\mathbf{V}=+\mathbf{0 . 5} \mathbf{V}$} & \multicolumn{2}{c}{$\mathbf{- 0 . 5} \mathbf{~ V}$} & \\
Molecule & $\log |\boldsymbol{J}|$ & $\boldsymbol{\sigma}_{\log }$ & $\log |\boldsymbol{J}|$ & $\boldsymbol{\sigma}_{\log }$ & $\boldsymbol{r}^{+\boldsymbol{a}}$ \\
\hline $\mathbf{6}_{(4,4)}$ & -0.56 & 0.22 & -0.83 & 0.22 & 1.86 \\
$\mathbf{7}_{(6,6)}$ & -1 & 0.23 & -1.417 & 0.43 & 2.61 \\
$\mathbf{8}_{(8,8)}$ & -1.43 & 0.46 & -1.69 & 0.54 & 1.82 \\
$\mathbf{9}_{(10,10)}$ & -1.95 & 0.47 & -2.05 & 0.42 & 1.26 \\
$\mathbf{1 0}_{(12,12)}$ & -2.88 & 0.5 & -3.13 & 0.57 & 1.78 \\
$a r^{+}=<|J(+1 \mathrm{~V})| /|J(-1 \mathrm{~V})|>$ & & & &
\end{tabular}


Table S10. Measured values of $\log |J|$ at $+1.0 \mathrm{~V}$ and $-1.0 \mathrm{~V}$ and the corresponding rectification ratios $(r+)$ for series I amides 5, 7 - 11.

\begin{tabular}{ccccccc} 
& \multicolumn{6}{c}{$\mathbf{V}=+\mathbf{1 . 0} \mathbf{V}=\mathbf{- 1 . 0} \mathbf{~ V}$} \\
Molecule & $\log |\boldsymbol{J}|$ & $\boldsymbol{\sigma}_{\log }$ & $\log |\boldsymbol{J}|$ & $\boldsymbol{\sigma}_{\log }$ & $\boldsymbol{r}^{+a}$ & $\boldsymbol{\sigma}_{r^{+}}$ \\
\hline $\mathbf{7}_{(6,6)}$ & -0.19 & 0.4 & -0.97 & 0.43 & 6.13 & 1.78 \\
$\mathbf{1 1}_{(8,4)}$ & -0.35 & 0.66 & -0.98 & 0.66 & 4.35 & 1.36 \\
$\mathbf{1 2}_{(10,2)}$ & -0.3 & 0.37 & -0.97 & 0.46 & 4.84 & 1.32 \\
$\mathbf{1 3}_{(11,1)}$ & -0.11 & 0.41 & -0.96 & 0.41 & 7.17 & 1.67 \\
$\mathbf{5}_{(12, \mathrm{H})}$ & -1.5 & 0.35 & -2.5 & 0.43 & 10.42 & 1.40 \\
$r_{r}^{+}=<|J(+1 \mathrm{~V})| /|J(-1 \mathrm{~V})|>$ & & & &
\end{tabular}

Table S11. Measured values of $\log |J|$ at $+0.5 \mathrm{~V}$ and $-0.5 \mathrm{~V}$ and the corresponding rectification ratios $(r+)$ for series I amides 5, 7 - 11.

\begin{tabular}{cccccc} 
& \multicolumn{2}{c}{$\mathbf{V}=+\mathbf{0 . 5} \mathbf{V}$} & \multicolumn{2}{c}{$\mathbf{- 0 . 5} \mathbf{~ V}$} & \\
Molecule & $\log |\boldsymbol{J}|$ & $\boldsymbol{\sigma}_{\log }$ & $\log |\boldsymbol{J}|$ & $\boldsymbol{\sigma}_{\log }$ & $\boldsymbol{r}^{+\boldsymbol{a}}$ \\
\hline $\mathbf{7}_{(6,6)}$ & -1 & 0.23 & -1.417 & 0.43 & 2.61 \\
$\mathbf{1 1}_{(8,4)}$ & -0.54 & 0.68 & -0.82 & 0.36 & 1.91 \\
$\mathbf{1 2}_{(10,2)}$ & -1.2176 & 0.45519 & -1.4076 & 0.48 & 1.55 \\
$\mathbf{1 3}_{(11,1)}$ & -1.16 & 0.7 & -1.42 & 0.42 & 1.82 \\
$\mathbf{5}_{(12, \mathrm{H})}$ & -2.84 & 0.30 & -3.1 & 0.38 & 1.82 \\
$a r^{+}=<|J(+1 \mathrm{~V})| /|J(-1 \mathrm{~V})|>$ & & & &
\end{tabular}


Table S12. Measured values of $\log |J|$ at $+1.0 \mathrm{~V}$ and $-1.0 \mathrm{~V}$ and the corresponding rectification ratios $(r+)$ for series II amides 5, 1014 - 18.

\begin{tabular}{|c|c|c|c|c|c|c|}
\hline \multirow[b]{2}{*}{ Molecule } & \multicolumn{2}{|c|}{$\mathrm{V}=+1.0 \mathrm{~V}$} & \multicolumn{2}{|c|}{$\mathrm{V}=-\mathbf{1 . 0} \mathrm{V}$} & \multirow[b]{2}{*}{$r^{+a}$} & \multirow[b]{2}{*}{$\sigma_{r^{+}}$} \\
\hline & $\log |J|$ & $\sigma_{\log }$ & $\log |J|$ & $\sigma_{\log }$ & & \\
\hline $\mathbf{5}_{(12, \mathrm{H})}$ & -1.5 & 0.35 & -2.5 & 0.43 & 10.42 & 1.4 \\
\hline $\mathbf{1 4}_{(12,2)}$ & -1.75 & 0.13 & -2.38 & 0.66 & 5.45 & 2.26 \\
\hline $15_{(12,4)}$ & -0.33 & 0.33 & -1.11 & 0.42 & 4.96 & 1.66 \\
\hline $\mathbf{1 6}_{(12,6)}$ & -0.1281 & 0.49 & -0.97 & 0.57 & 7.28 & 1.44 \\
\hline $17_{(12,8)}$ & -0.67 & 0.36 & -1.52 & 0.34 & 6.87 & 1.73 \\
\hline $\mathbf{1 8}_{(12,10)}$ & -1 & 0.14 & -1.71 & 0.39 & 6.13 & 2.18 \\
\hline $\mathbf{1 0}_{(12,12)}$ & -1.86 & -0.1 & -2.86 & 0.08 & 11.22 & 2.27 \\
\hline
\end{tabular}

Table S13. Measured values of $\log |J|$ at $+0.5 \mathrm{~V}$ and $-0.5 \mathrm{~V}$ and the corresponding rectification ratios $(r+)$ for series II amides 5, $1014-18$.

\begin{tabular}{cccccc} 
& \multicolumn{2}{c}{$\mathbf{V}=+\mathbf{0 . 5} \mathbf{V}$} & \multicolumn{2}{c}{$\mathbf{~}=\mathbf{- 0 . 5} \mathbf{~}$} & \\
Molecule & $\log |\boldsymbol{J}|$ & $\boldsymbol{\sigma}_{\log }$ & $\log |\boldsymbol{J}|$ & $\boldsymbol{\sigma}_{\log }$ & $\boldsymbol{r}^{+\boldsymbol{a}}$ \\
\hline $\mathbf{5}_{(12, \mathrm{H})}$ & -2.84 & 0.30 & -3.1 & 0.38 & 1.82 \\
$\mathbf{1 4}_{(12,2)}$ & -2.37 & 0.66 & -2.6 & 0.73 & 1.70 \\
$\mathbf{1 5}_{(12,4)}$ & -1.37 & 0.42 & -1.62 & 0.45 & 1.78 \\
$\mathbf{1 6}_{(12,6)}$ & -1.15 & 0.58 & -1.44 & 0.61 & 1.95 \\
$\mathbf{1 7}_{(12,8)}$ & -1.77 & 0.4 & -2.02 & 0.37 & 1.78 \\
$\mathbf{1 8}_{(12,10)}$ & -2.1 & 0.44 & -2.31 & 0.43 & 1.62 \\
$\mathbf{1 0}_{(12,12)}$ & -2.88 & 0.5 & -3.13 & 0.57 & 1.78
\end{tabular}


A

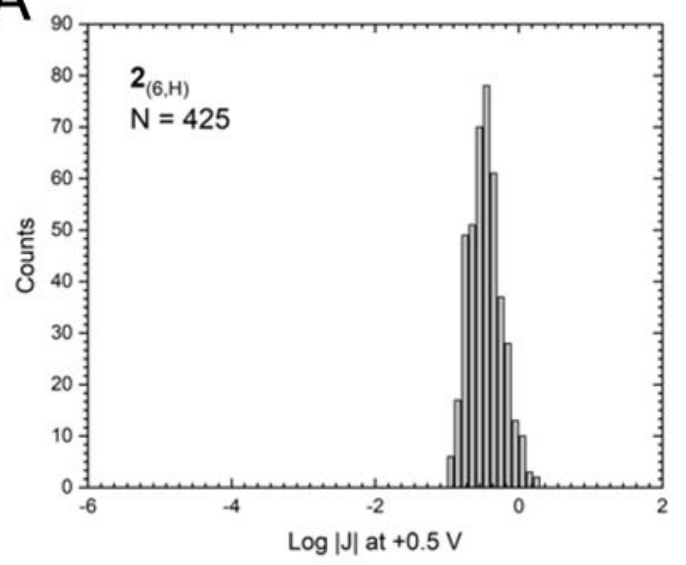

C

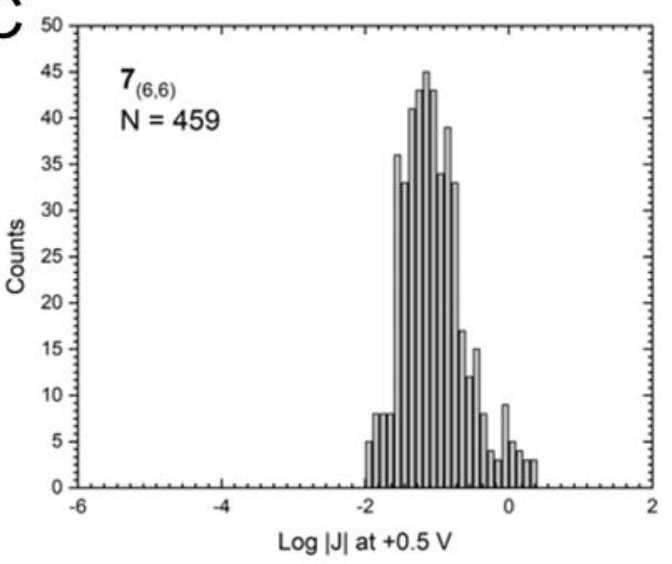

$E$

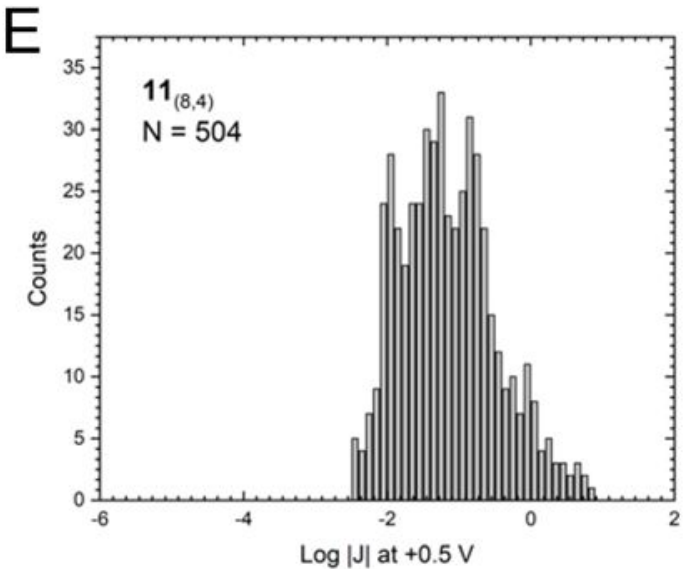

B

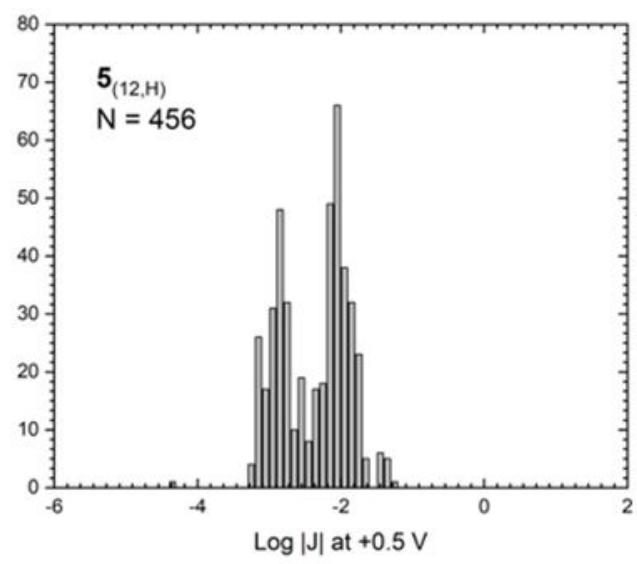

D

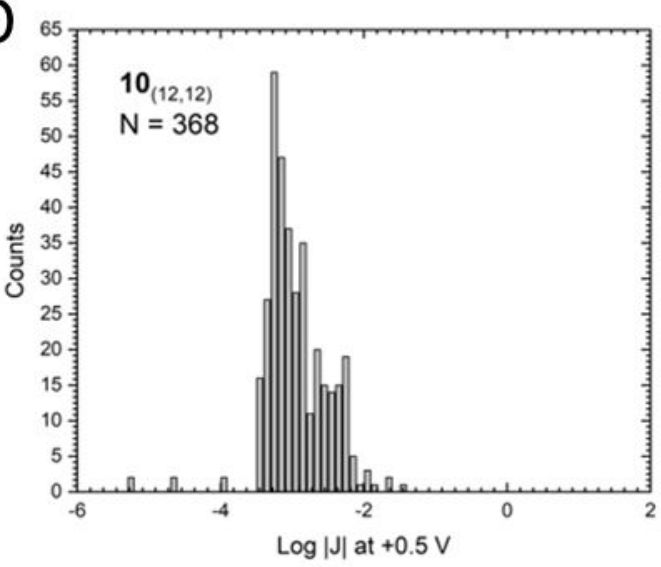

$F$

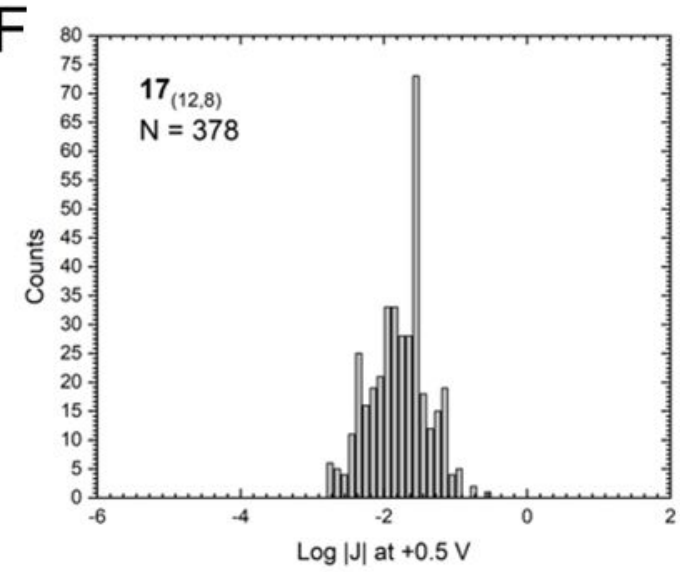

Figure S6. Plots showing histograms of current density measurements performed with the EGaIn junction for a selected set of SAMS including (A) $2_{(6, \mathrm{H})}$, (B) $5_{(12, \mathrm{H})},(\mathrm{C}) 7_{(6,6)}$, (D) $10_{(12,12)}$, (E) $11_{(8,4)},(\mathrm{F}) 17_{(12,8)}$. 


\section{S10. Summary of simulation data}

Table S14. Selected data from the simulations.

\begin{tabular}{|c|c|c|c|c|c|c|c|}
\hline & $\begin{array}{c}\text { Simulated } \\
\text { Thickness } \\
(\AA)\end{array}$ & $\begin{array}{l}\text { Standard } \\
\text { Deviation }\end{array}$ & $\begin{array}{l}\text { Predicted } \\
\text { Surface } \\
\text { Coverage } \\
\left(\mathbf{m o l} / \mathrm{cm}^{2}\right)\end{array}$ & $\begin{array}{c}f_{(\theta \text { trans })} \\
\left(\frac{\theta(\text { trans })}{\theta(\text { trans })+\theta(\text { gauche })}\right)\end{array}$ & $f_{\text {(bent) }}$ & $\begin{array}{l}\text { simulated } \\
\% \text { trans } \\
\text { CCCC }\end{array}$ & $\begin{array}{c}\text { simulated } \\
\% \text { trans } \\
\text { CNCC }\end{array}$ \\
\hline$\overline{\mathbf{1}_{(4, \mathrm{H})}}$ & 9.12 & 0.65 & 1 & & 0 & & \\
\hline $\boldsymbol{2}_{(6, \mathrm{H})}$ & 12 & 0.57 & 1 & 0.87 & 0 & 0.87 & 0.68 \\
\hline $\mathbf{3}_{(8, \mathrm{H})}$ & 14.09 & 0.65 & 1 & 0.93 & 0 & 0.93 & 0.84 \\
\hline $\mathbf{4}_{(10, \mathrm{H})}$ & 16.09 & 0.74 & 1 & 0.97 & 0 & 0.97 & 0.91 \\
\hline $\mathbf{5}_{(12, \mathrm{H})}$ & 18.13 & 0.65 & 1 & 0.99 & 0 & 0.99 & 1 \\
\hline $\mathbf{6}_{(4,4)}$ & 9.307 & 0.78 & 0.79 & & & & \\
\hline $7_{(6,6)}$ & 11.92 & 0.74 & 0.758 & 0.74 & 0.21 & 0.74 & 0.66 \\
\hline $\mathbf{8}_{(8,8)}$ & 14.34 & 0.78 & 0.64 & 0.81 & 0.14 & 0.81 & 0.67 \\
\hline $\mathbf{9}_{(10,10)}$ & 17 & 0.82 & 0.65 & 0.88 & 0.08 & 0.88 & 0.62 \\
\hline $\mathbf{1 0}_{(12,12)}$ & 19.15 & 0.84 & 0.59 & 0.88 & 0.06 & 0.88 & 0.64 \\
\hline $\mathbf{1 1}_{(8,4)}$ & 13.767 & 0.512 & 0.863 & 0.78 & 0.58 & 0.78 & 0.72 \\
\hline $\mathbf{1 2}_{(10,2)}$ & 16.283 & 1.14 & 0.887 & 0.91 & 0.35 & 0.91 & 0.92 \\
\hline $\mathbf{1 3}_{(11,1)}$ & 17.74 & 0.93 & 1 & 0.98 & 0 & 0.98 & 0.96 \\
\hline $\mathbf{1 4}_{(12,2)}$ & 18.56 & 1.3 & 0.91 & 0.93 & 0.5 & 0.99 & 1 \\
\hline $\mathbf{1 5}_{(12,4)}$ & 18 & 1.56 & 0.76 & 0.82 & 0.6 & 0.93 & 0.93 \\
\hline $1 \mathbf{6}_{(12,6)}$ & 19.43 & 2.1 & 0.73 & 0.83 & 0.32 & 0.82 & 0.72 \\
\hline $\mathbf{1 7}_{(12,8)}$ & 19.07 & 0.97 & 0.69 & 0.86 & 0.15 & 0.83 & 0.67 \\
\hline $\mathbf{1 8}_{(12,10)}$ & 18.72 & 1.08 & 0.61 & 0.86 & 0.17 & 0.86 & 0.64 \\
\hline
\end{tabular}



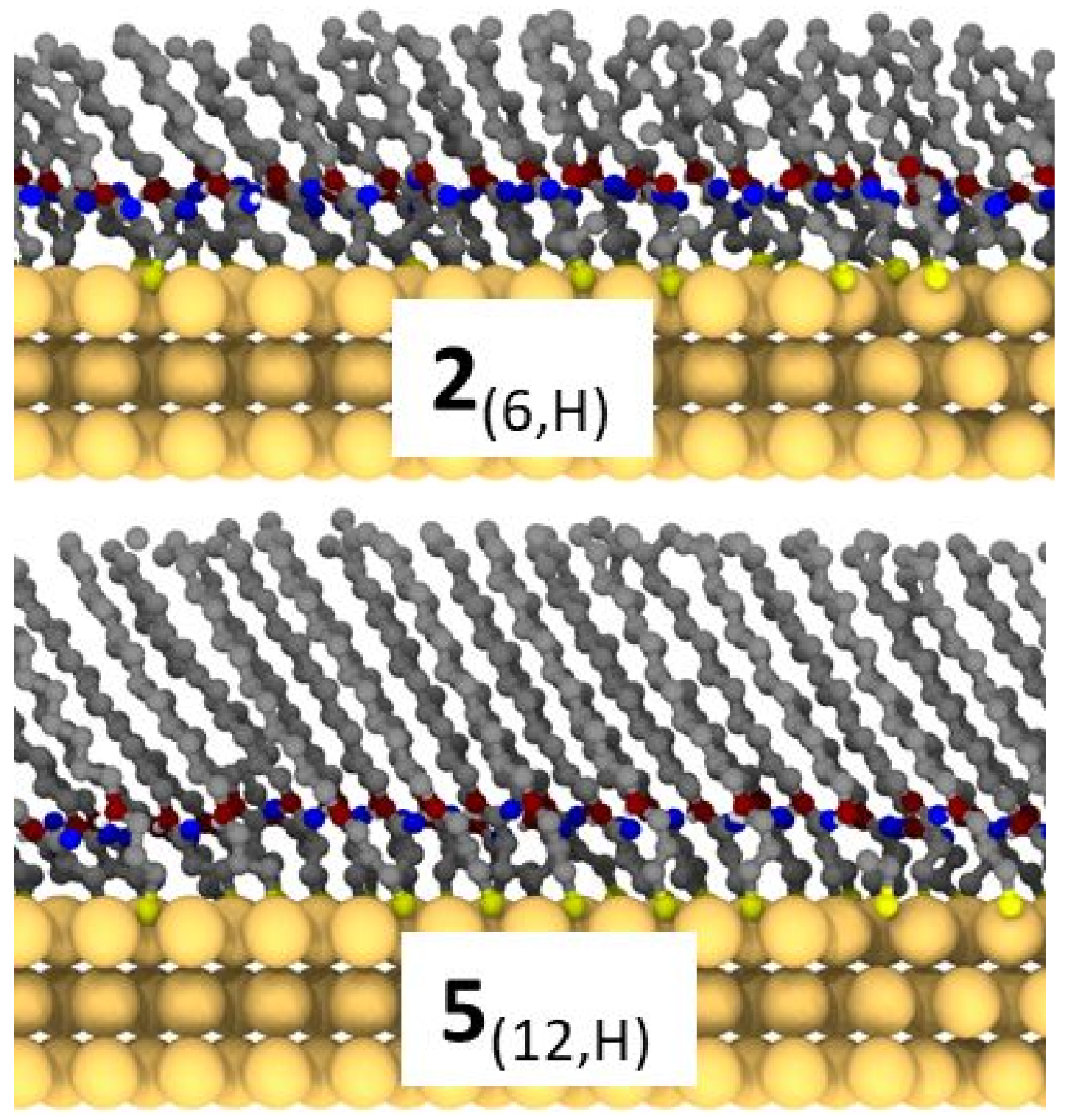

Figure S7. Representative structures of the monolayers obtained from molecular dynamics simulations for Control Series I. 

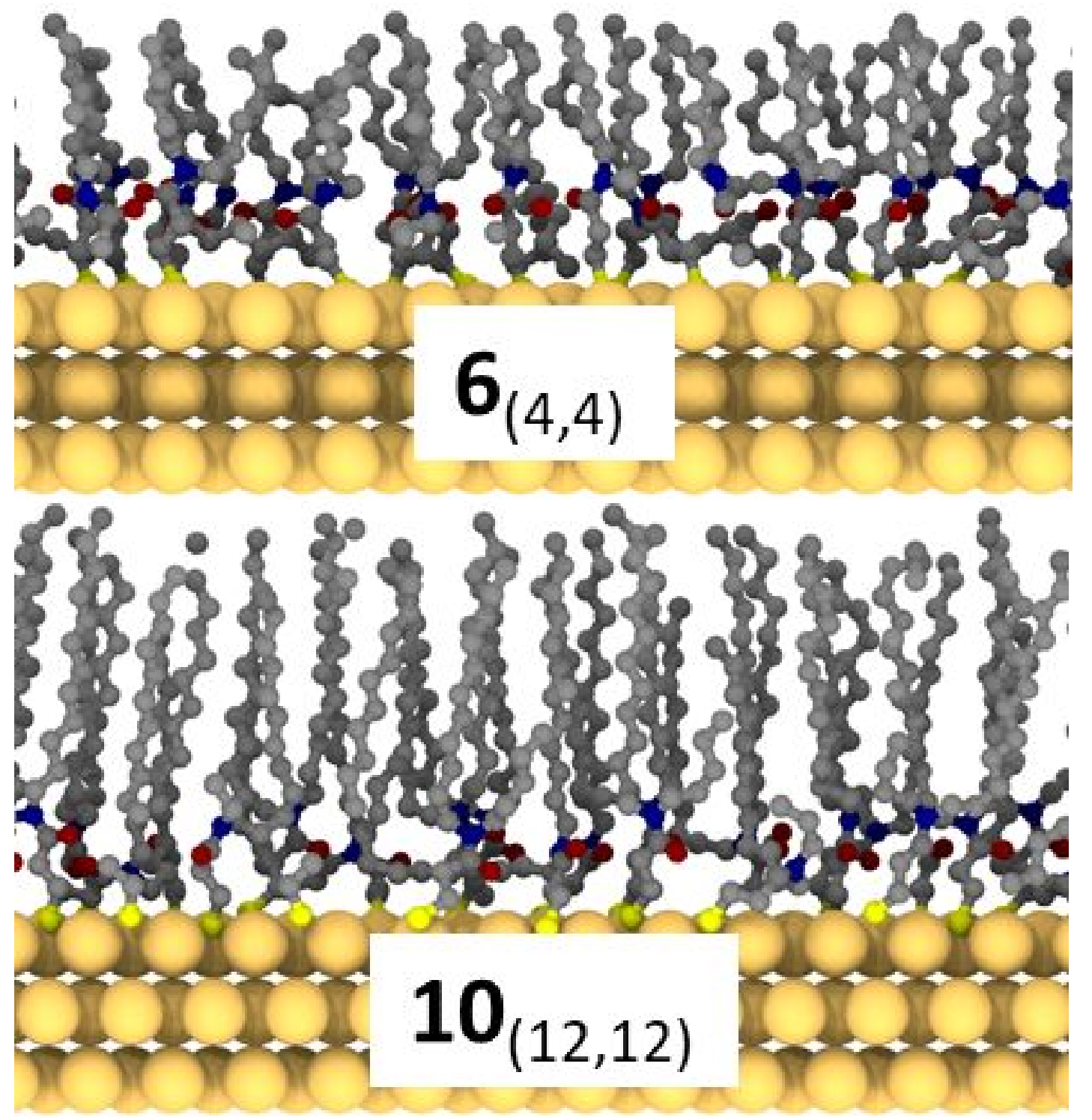

Figure S8. Representative structures of the monolayers obtained from molecular dynamics simulations for Control Series II. 

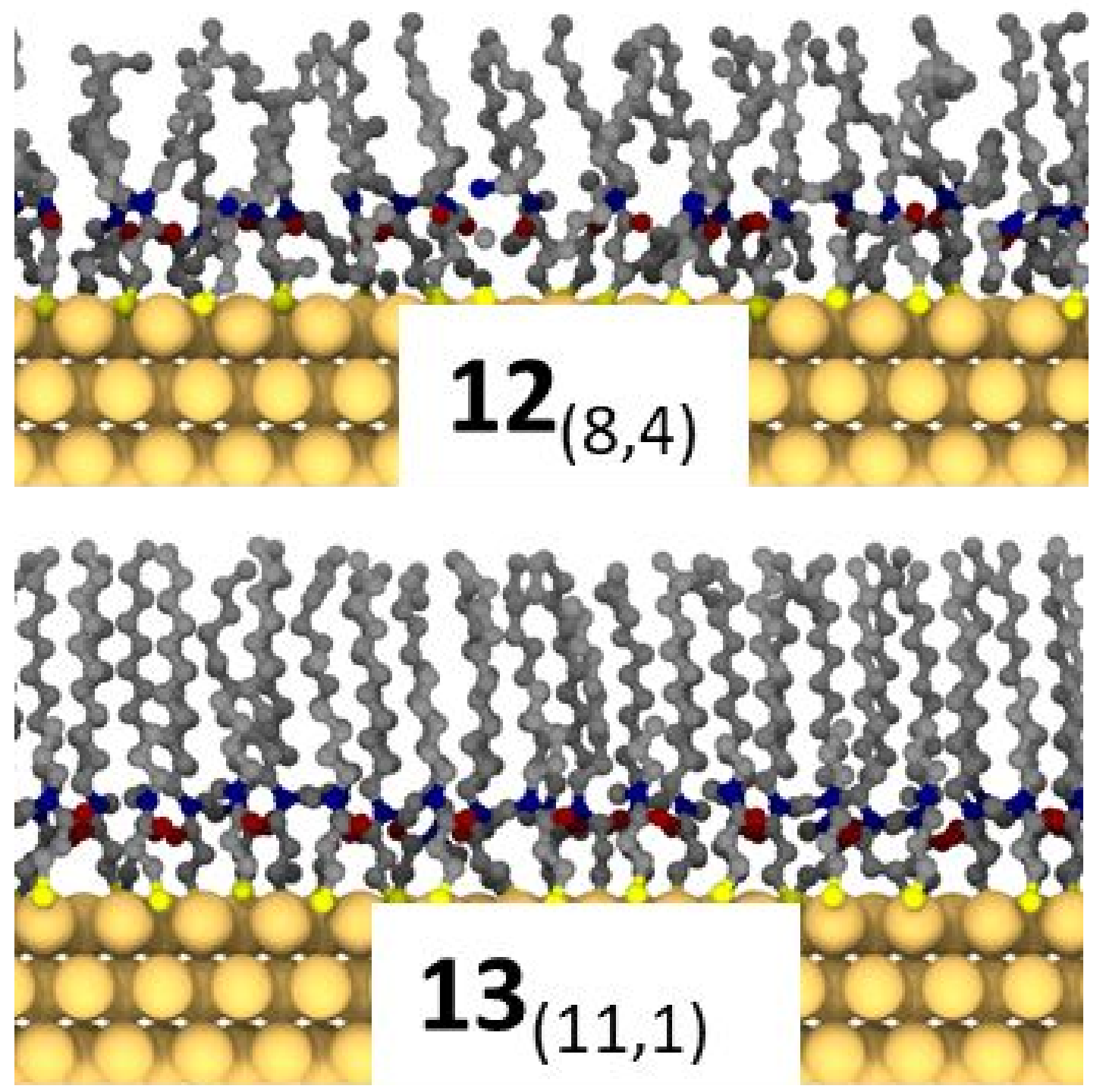

Figure S9. Representative structures of the monolayers obtained from molecular dynamics simulations for Disordered Series I. 

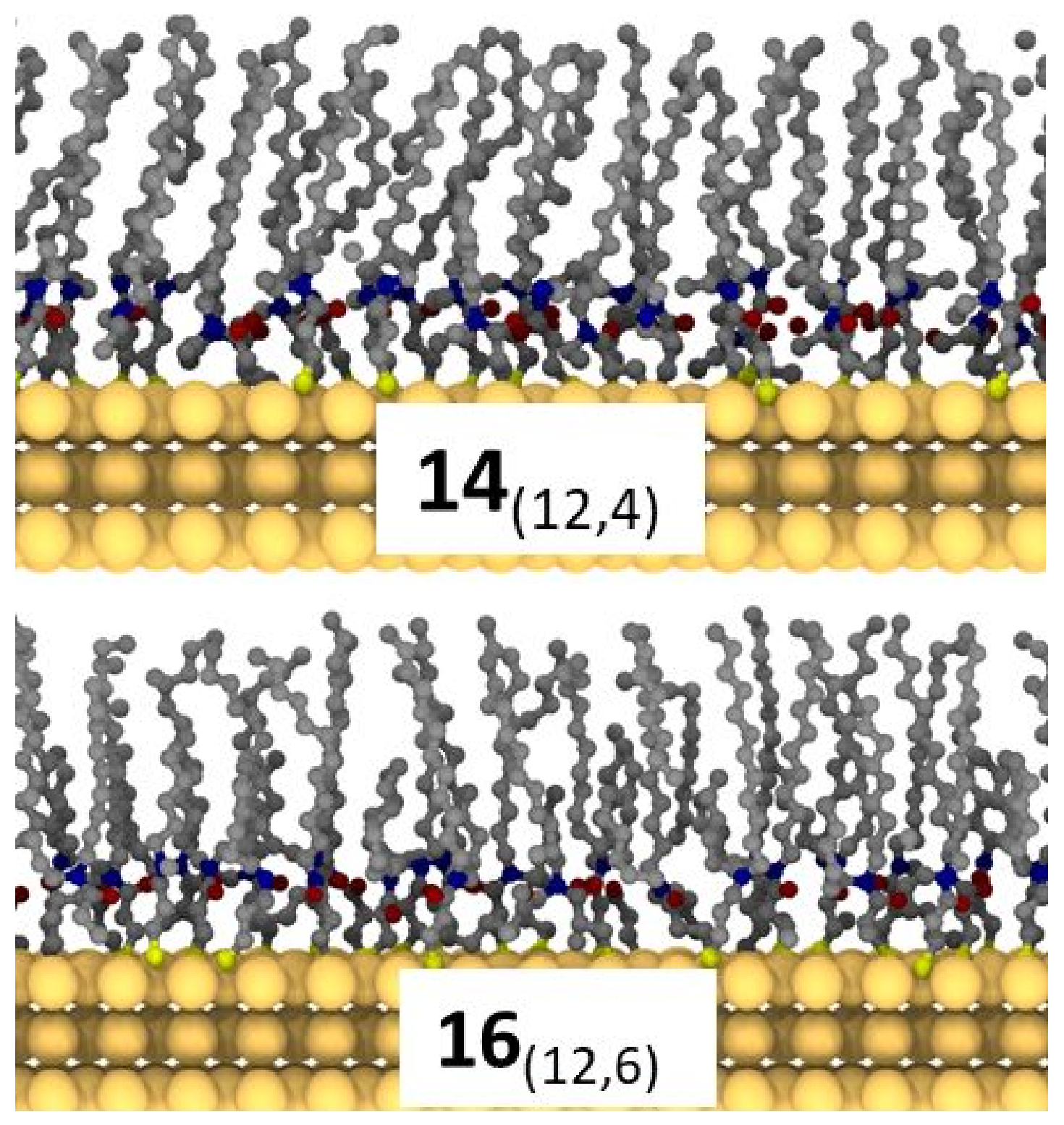

Figure S10. Representative structures of the monolayers obtained from molecular dynamics simulations for Disordered Series II. The empty spaces in these cross-sectional images are an artifact of the way the system is visualized (i.e. without using a space-filling model, and with atoms being cutoff due to the cross-sectioning procedure.) 


\section{S11. Synthesis}

A general synthetic procedure, which was applied in the synthesis of all molecules used in this work, is shown here. The method of purification, yield, and characterization of each individual intermediate is present for each individual molecule, in the following section. Unless otherwise specified, all reactions were performed under an inert atmosphere of dry Argon.

General 3-step procedure for the synthesis of non-symmetrical amines.

*symmetric dialkyl amines and primary amine were commercially available.

Step 1.

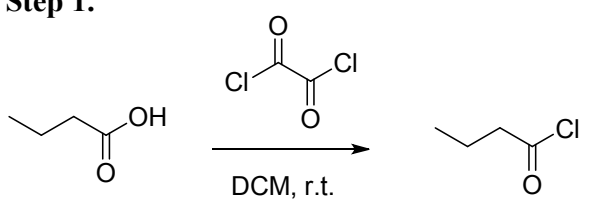

Step 2.

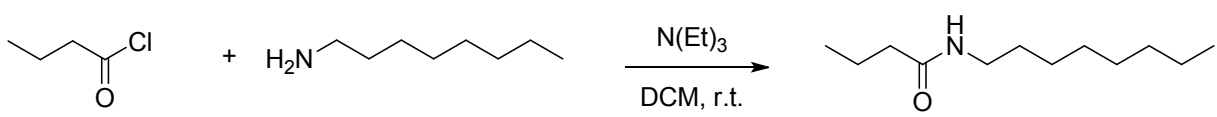

Step 3.

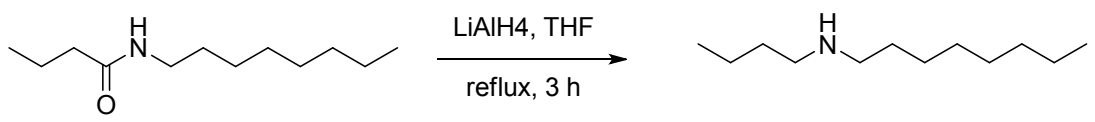

\section{N-pentylbutyramide (Steps 1 and 2)}

Butyric acid (2 mmol, 1 eq.) was dissolved in dichloromethane $(10 \mathrm{~mL})$. Oxalyl chloride (4.4

mmol, 2.2 eq.) was then added dropwise. The reaction was stirred at room temperature for 3 hours, at which point the reaction mixture was concentrated in vacuo to afford the corresponding butyric acid chloride as a colorless oil. Without further purification, the acid chloride was dissolved in dichloromethane (10 mL). Octylamine ( $2.2 \mathrm{mmol}, 1.1 \mathrm{eq}$.), followed by triethylamine $(2.2 \mathrm{mmol}, 1.1)$, were then added, dropwise, to the solution. The reaction was stirred at room temperature for 16 hours. The reaction was quenched with $5 \mathrm{~mL} 1$ of $\mathrm{M} \mathrm{HCl}$. The 
reaction mixture was then transferred to a separatory funnel where it was extracted twice with dichloromethane $(10 \mathrm{~mL})$ and washed once with brine $(10 \mathrm{~mL})$. The organic layer was dried over $\mathrm{MgSO}_{4}$ and concentrated in vacuo to afford a light yellow oil. The product was either purified by silica gel column chromatography with a hexane:ehthyl acetate mixture $(4: 1 ; \mathrm{v}: \mathrm{v})$, or used in its crude form without further purification.

\section{N-butyloctan-1-amine (Step 3)}

N-pentylbutyramide (2 mmol, 1 eq.) was dissolved in tetrahydrofuran $(5 \mathrm{~mL})$, and added dropwise to a suspension of $\mathrm{LiAlH}_{4}(6 \mathrm{mmol}, 3$ eq. $)$ in tetrahydrofuran $(10 \mathrm{~mL})$, at $0{ }^{\circ} \mathrm{C}$. The reaction was heated to $60^{\circ} \mathrm{C}$ for 4 hours. The reaction mixture was cooled to room temperature, quenched with $\mathrm{H}_{2} \mathrm{O}(5 \mathrm{~mL})$, and filtered. The resulting liquid was treated with $1 \mathrm{M} \mathrm{HCl}$ until the $\mathrm{pH}$ of solution was $\mathrm{pH}=3$. The reaction mixture was then transferred to a separatory funnel, and washed once with ethyl acetate $(10 \mathrm{~mL})$. The aqueous layer was then treated with $1 \mathrm{M} \mathrm{NaOH}$ until $\mathrm{pH}=8$, and the product was extracted three times with ethyl acetate $(10 \mathrm{~mL})$. The organic layer was dried over $\mathrm{MgSO}_{4}$ and concentrated in vacuo to afford a clear oil.

\section{Synthesis of 3,3'-disulfanediyldipropanoyl chloride}

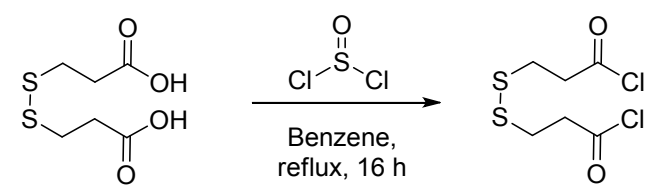

3,3-dithiopropionic acid ( $2 \mathrm{mmol}, 1$ eq.) was dissolved in benzene $(20 \mathrm{~mL})$. Thionyl chloride (10 mmol, 5 eq.) was added dropwise, to the solution. The reaction mixture was heated to reflux for 16 hours. The mixture was concentrated in vacuo to yield pure 3,3-dithiopropionyl chloride as a clear oil in $>95 \%$ yield. 
General synthetic procedure for the synthesis of linear, branched, and cyclic amides from amines.

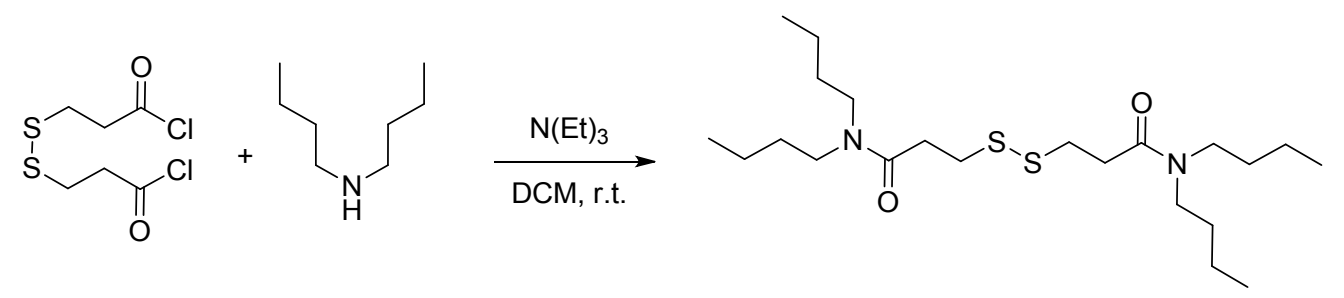

A primary or secondary amine (1.05 mmol, 2.1 eq.) was added drop-wise to a solution of propionyl chloride ( $0.5 \mathrm{mmol}, 1$ eq.) in dichloromethane, followed by the drop-wise addition of triethylamine (1.05 mmol, 2.1 eq.). The reaction mixture was stirred at room temperature for 16 hours. The reaction mixture was concentrated in vacuo, and partitioned between ethyl acetate (10 $\mathrm{mL})$ and $1 \mathrm{M} \mathrm{HCl}(5 \mathrm{~mL})$. The aqueous phase was extracted twice more with ethyl acetate (10 $\mathrm{mL}$ ). The mixture was concentrated in vacuo before purification by silica gel chromatography.

\section{Purification procedure and identification of all products and intermediates.}

\section{Linear Amides}

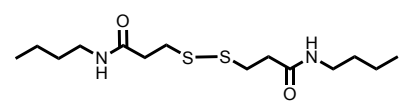

\section{3,3'-disulfanediylbis(N-butylpropanamide)}

The product was purified by silica gel column chromatography with a dichloromethane:methanol mixture (95:5; v:v), to afford a white solid. ${ }^{1} \mathrm{H}-\mathrm{NMR}\left(500 \mathrm{MHz}, \mathrm{CDCl}_{3}\right): \delta=5.94$ (bs, $\left.2 \mathrm{H}\right), 3.26$ $-3.30(\mathrm{~m}, 2 \mathrm{H}), 2.99-3.02(\mathrm{~m}, 4 \mathrm{H}), 2.57-2.60(\mathrm{~m}, 2 \mathrm{H}), 1.49-1.55(\mathrm{~m}, 4 \mathrm{H}) 1.34-1.41(\mathrm{~m}$, 4H), $0.93-0.96(\mathrm{~m}, 6 \mathrm{H}) .{ }^{13} \mathrm{C}-\mathrm{NMR}\left(126 \mathrm{MHz}, \mathrm{CDCl}_{3}\right): \delta=170.8,39.4,35.8,34.3,31.6,20.1$, 13.7. HRMS (ESI): $\mathrm{m} / \mathrm{z}$ calcd for $\mathrm{C}_{14} \mathrm{H}_{28} \mathrm{~N}_{2} \mathrm{O}_{2} \mathrm{~S}_{2}\left[\mathrm{M}+\mathrm{H}^{+}\right]^{+}: 321.1665$, found: 321.1663 


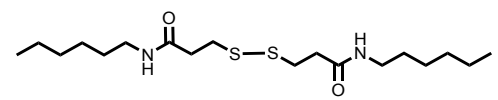

\section{3,3'-disulfanediylbis(N-hexylpropanamide)}

The product was purified by silica gel column with a dichloromethane:methanol mixture (95:5; v:v), to afford a white solid. ${ }^{1} \mathrm{H}-\mathrm{NMR}\left(500 \mathrm{MHz}, \mathrm{CDCl}_{3}\right): \delta=6.30$ (bs, $\left.2 \mathrm{H}\right), 3.23(\mathrm{q}, 4 \mathrm{H}), 2.98$ $(\mathrm{t}, 4 \mathrm{H}, \mathrm{J}=7.03 \mathrm{~Hz}), 2.57(\mathrm{t}, 4 \mathrm{H}, 7.04), 1.47-1.53(\mathrm{~m}, 4 \mathrm{H}), 1.25-1.34(\mathrm{~m}, 12 \mathrm{H}), 0.87(\mathrm{t}, 6 \mathrm{H}, \mathrm{J}$ $=6.81 \mathrm{~Hz}) \cdot{ }^{13} \mathrm{C}-\mathrm{NMR}\left(126 \mathrm{MHz}, \mathrm{CDCl}_{3}\right): \delta=171.0,39.7,35.8,34.4,31.5,29.5,26.6,22.5,14.0$. HRMS (ESI): $\mathrm{m} / \mathrm{z}$ calcd for $\mathrm{C}_{18} \mathrm{H}_{38} \mathrm{~N}_{2} \mathrm{O}_{2} \mathrm{~S}_{2}\left[\mathrm{M}+\mathrm{H}^{+}\right]^{+}: 377.2291$, found: 377.2285 .

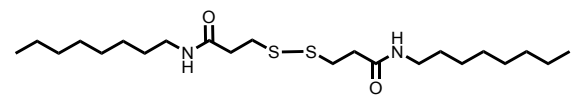

\section{3,3'-disulfanediylbis(N-octylpropanamide):}

The product was purified by silica gel column chromatography with a dichloromethane:methanol mixture (95:5; v:v), to afford a white solid. ${ }^{1} \mathrm{H}-\mathrm{NMR}\left(500 \mathrm{MHz}, \mathrm{CDCl}_{3}\right)$ : $\delta=6.36$ (bs, $\left.2 \mathrm{H}\right), 3.26$ - $3.30(\mathrm{~m}, 4 \mathrm{H}), 3.00(\mathrm{t}, 4 \mathrm{H}, \mathrm{J}=7.01 \mathrm{~Hz}), 2.63(\mathrm{t}, 4 \mathrm{H}, \mathrm{J}=7.01 \mathrm{~Hz}), 1.51-1.57(\mathrm{~m}, 4 \mathrm{H}) 1.28-$ $1.31(\mathrm{~m}, 20 \mathrm{H}), 0.89(\mathrm{t}, 6 \mathrm{H}, \mathrm{J}=6.95) .{ }^{13} \mathrm{C}-\mathrm{NMR}\left(126 \mathrm{MHz}, \mathrm{CDCl}_{3}\right): \delta=171.2,40.0,35.6,34.4$, 31.8, 29.5, 29.3, 29.2, 26.9, 22.6, 14.1. HRMS (ESI): $\mathrm{m} / \mathrm{z}$ calcd for $\mathrm{C}_{22} \mathrm{H}_{44} \mathrm{~N}_{2} \mathrm{O}_{2} \mathrm{~S}_{2}\left[\mathrm{M}+\mathrm{H}^{+}\right]^{+}$: 433.2917, found: 433.2921 .

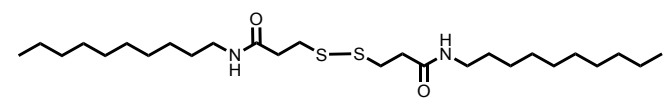

\section{3,3'-disulfanediylbis(N-decylpropanamide)}

The product was purified by silica gel column chromatography with a dichloromethane:methanol mixture (95:5; v:v), to afford a white solid. ${ }^{1} \mathrm{H}-\mathrm{NMR}\left(500 \mathrm{MHz}, \mathrm{CDCl}_{3}\right)$ : $\delta=6.39$ (bs, $\left.2 \mathrm{H}\right), 3.25$ $-3.29(\mathrm{~m}, 4 \mathrm{H}), 3.02(\mathrm{t}, 4 \mathrm{H}, \mathrm{J}=6.99 \mathrm{~Hz}), 2.62(\mathrm{t}, 4 \mathrm{H}, \mathrm{J}=6.99 \mathrm{~Hz}), 1.51-1.56(\mathrm{~m}, 4 \mathrm{H}) 1.27(\mathrm{~s}$, 
$28 \mathrm{H}), 0.89(\mathrm{t}, 6 \mathrm{H}, \mathrm{J}=6.78 \mathrm{~Hz}) \cdot{ }^{13} \mathrm{C}-\mathrm{NMR}\left(126 \mathrm{MHz}, \mathrm{CDCl}_{3}\right): \delta=171.3,40.0,35.6,34.4,31.9$, 29.6, 29.5, 29.3, 29.3, 27.0, 22.7, 14.1. HRMS (ESI): $\mathrm{m} / \mathrm{z}$ calcd for $\mathrm{C}_{26} \mathrm{H}_{52} \mathrm{~N}_{2} \mathrm{O}_{2} \mathrm{~S}_{2}\left[\mathrm{M}+\mathrm{H}^{+}\right]^{+}$: 489.3543, found: 489.3546 .

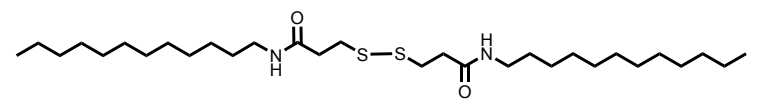

\section{3,3'-disulfanediylbis(N-dodecylpropanamide)}

The product was purified by silica gel column chromatography with a dichloromethane:methanol mixture (95:5; v:v), to afford a white solid. ${ }^{1} \mathrm{H}-\mathrm{NMR}\left(500 \mathrm{MHz}, \mathrm{CDCl}_{3}\right): \delta=6.54$ (bs, $\left.2 \mathrm{H}\right), 3.27$ $-3.31(\mathrm{~m}, 4 \mathrm{H}), 3.05(\mathrm{t}, 4 \mathrm{H}, \mathrm{J}=6.87 \mathrm{~Hz}), 2.66(\mathrm{t}, 4 \mathrm{H}, \mathrm{J}=6.87 \mathrm{~Hz}), 1.55-1.58(\mathrm{~m}, 4 \mathrm{H}) 1.27(\mathrm{~s}$, $36 \mathrm{H}), 0.90(\mathrm{t}, 6 \mathrm{H}, \mathrm{J}=7.13 \mathrm{~Hz}) .{ }^{13} \mathrm{C}-\mathrm{NMR}\left(126 \mathrm{MHz}, \mathrm{CDCl}_{3}\right): \delta=170.8,39.8,35.8,34.3,31.9$, 29.7, 29.6, 29.6, 29.6, 29.4, 29.3, 29.3, 27.0, 22.7, 14.1. HRMS HRMS (ESI): m/z calcd for $\mathrm{C}_{30} \mathrm{H}_{60} \mathrm{~N}_{2} \mathrm{O}_{2} \mathrm{~S}_{2}\left[\mathrm{M}+\mathrm{H}^{+}\right]^{+}:$545.4169, found: 545.4162

\section{Branched Amides, Series I}

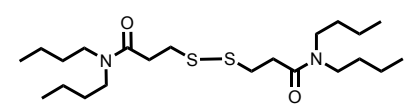

3,3'-disulfanediylbis(N-butylpropanamide)

The product was purified by silica gel column chromatography with a mixture of hexanes:ethyl acetate $(4: 1 ; \mathrm{v}: \mathrm{v})$ to afford a clear oil. ${ }^{1} \mathrm{H}-\mathrm{NMR}\left(500 \mathrm{MHz}, \mathrm{CDCl}_{3}\right): \delta=3.30(\mathrm{t}, 4 \mathrm{H}, \mathrm{J}=7.62 \mathrm{~Hz})$, $3.24(\mathrm{t}, 4 \mathrm{H}, \mathrm{J}=7.76 \mathrm{~Hz}), 2.98(\mathrm{t}, 4 \mathrm{H}, \mathrm{J}=7.17 \mathrm{~Hz}), 2.75(\mathrm{t}, 4 \mathrm{H}, \mathrm{J}=7.17 \mathrm{~Hz}), 1.49-1.60(\mathrm{~m}$, $8 \mathrm{H}), 1.26-1.34(\mathrm{~m}, 24 \mathrm{H}), 0.88-0.93(\mathrm{~m}, 12 \mathrm{H}) .{ }^{13} \mathrm{C}-\mathrm{NMR}\left(126 \mathrm{MHz}, \mathrm{CDCl}_{3}\right): \delta=170.3,48.0$, 
46.2, 33.6, 32.8, 31.6, 31.5, 29.1, 27.7, 26.7, 26.6, 22.6, 14.0. HRMS (ESI): $\mathrm{m} / \mathrm{z}$ calcd for $\mathrm{C}_{22} \mathrm{H}_{44} \mathrm{~N}_{2} \mathrm{O}_{2} \mathrm{~S}_{2}\left[\mathrm{M}+\mathrm{H}^{+}\right]^{+}:$433.2917, found: 433.2922 .

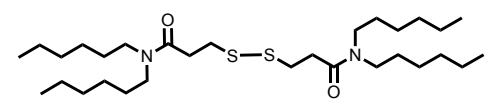

3,3'-disulfanediylbis(N,N-hexylpropanamide)

The product was purified by silica gel column chromatography with a mixture of hexanes:ethyl acetate $(4: 1 ; \mathrm{v}: \mathrm{v})$ to afford a clear oil. ${ }^{1} \mathrm{H}-\mathrm{NMR}\left(500 \mathrm{MHz}, \mathrm{CDCl}_{3}\right): \delta=3.29(\mathrm{t}, 4 \mathrm{H}, \mathrm{J}=7.62 \mathrm{~Hz})$, $3.22(\mathrm{t}, 4 \mathrm{H}, \mathrm{J}=7.76 \mathrm{~Hz}), 2.96(\mathrm{t}, 4 \mathrm{H}, \mathrm{J}=7.17 \mathrm{~Hz}), 2.72(\mathrm{t}, 4 \mathrm{H}, \mathrm{J}=7.17 \mathrm{~Hz}), 1.47-1.59(\mathrm{~m}$, $8 \mathrm{H}), 1.26-1.30(\mathrm{~m}, 24 \mathrm{H}), 0.86-0.91(\mathrm{~m}, 12 \mathrm{H}) .{ }^{13} \mathrm{C}-\mathrm{NMR}\left(126 \mathrm{MHz}, \mathrm{CDCl}_{3}\right): \delta=170.3,48.0$, 46.2, 33.6, 32.8, 31.6, 31.5, 29.1, 27.7, 26.7, 26.6, 22.6, 14.0. HRMS (ESI): $\mathrm{m} / \mathrm{z}$ calcd for $\mathrm{C}_{30} \mathrm{H}_{60} \mathrm{~N}_{2} \mathrm{O}_{2} \mathrm{~S}_{2}\left[\mathrm{M}+\mathrm{H}^{+}\right]^{+}: 545.4169$ found: 545.4159 .

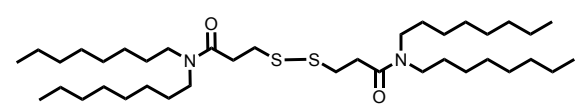

3,3'-disulfanediylbis(N,N-dioctylpropanamide)

The product was purified by silica gel column chromatography with a mixture of hexanes:ethyl acetate $(4: 1 ; \mathrm{v}: \mathrm{v})$ to afford a clear oil. ${ }^{1} \mathrm{H}-\mathrm{NMR}\left(500 \mathrm{MHz}, \mathrm{CDCl}_{3}\right): \delta=3.29(\mathrm{t}, 4 \mathrm{H}, \mathrm{J}=7.71 \mathrm{~Hz})$, $3.23(\mathrm{t}, 4 \mathrm{H}, \mathrm{J}=7.71 \mathrm{~Hz}), 2.97(\mathrm{t}, 4 \mathrm{H}, \mathrm{J}=7.12 \mathrm{~Hz}), 2.75(\mathrm{t}, 4 \mathrm{H}, \mathrm{J}=7.12 \mathrm{~Hz}), 1.49-1.57(\mathrm{~m}$, $8 \mathrm{H}), 1.23-1.30(\mathrm{~m}, 40 \mathrm{H}), 0.86-0.90(\mathrm{~m}, 12 \mathrm{H}) .{ }^{13} \mathrm{C} \mathrm{NMR}\left(126 \mathrm{MHz}, \mathrm{CDCl}_{3}\right) \delta$ 170.1, 48.2, 46.0, 33.5, 32.8, 31.8, 31.8, 29.4, 29.3, 29.3, 29.2, 29.1, 27.8, 27.1, 26.9, 22.6, 14.1. HRMS (ESI): $\mathrm{m} / \mathrm{z}$ calcd for $\mathrm{C}_{38} \mathrm{H}_{76} \mathrm{~N}_{2} \mathrm{O}_{2} \mathrm{~S}_{2}\left[\mathrm{M}+\mathrm{H}^{+}\right]^{+}: 657.5421$, found: 657.5428 . 


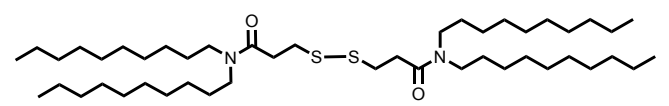

3,3'-disulfanediylbis(N,N-didecylpropanamide)

The product was purified by silica gel column chromatography with a mixture of hexanes:ethyl acetate $(4: 1 ; \mathrm{v}: \mathrm{v})$ to afford a clear oil. ${ }^{1} \mathrm{H}-\mathrm{NMR}\left(500 \mathrm{MHz}, \mathrm{CDCl}_{3} \delta=3.30(\mathrm{t}, 4 \mathrm{H}, \mathrm{J}=7.30 \mathrm{~Hz})\right.$, $3.23(\mathrm{t}, 4 \mathrm{H}, \mathrm{J}=7.30 \mathrm{~Hz}), 2.97(\mathrm{t}, 4 \mathrm{H}, \mathrm{J}=7.46 \mathrm{~Hz}), 2.75(\mathrm{t}, 4 \mathrm{H}, \mathrm{J}=7.46 \mathrm{~Hz}), 1.49-1.57(\mathrm{~m}$, $8 \mathrm{H}), 1.23-1.30(\mathrm{~m}, 56 \mathrm{H}), 0.86-0.90(\mathrm{~m}, 12 \mathrm{H}) .{ }^{13} \mathrm{C} \mathrm{NMR}\left(126 \mathrm{MHz}, \mathrm{CDCl}_{3}\right) \delta=170.3,48.0$, $46.1,33.6,32.8,31.8,31.8,29.4,29.3,29.3,29.2,29.1,27.8,27.1,26.9,22.6,14.1$. HRMS (ESI): $\mathrm{m} / \mathrm{z}$ calcd for $\mathrm{C}_{46} \mathrm{H}_{92} \mathrm{~N}_{2} \mathrm{O}_{2} \mathrm{~S}_{2}\left[\mathrm{M}+\mathrm{H}^{+}\right]^{+}:$768.6673, found:769.6646.

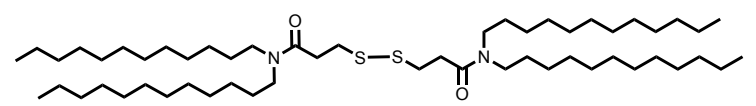

3,3'-disulfanediylbis(N,N-didodecylpropanamide)

The product was purified by silica gel column chromatography with a mixture of hexanes:ethyl acetate $(4: 1 ; \mathrm{v}: \mathrm{v})$ to afford a clear oil. ${ }^{1} \mathrm{H}-\mathrm{NMR}\left(500 \mathrm{MHz}, \mathrm{CDCl}_{3} \delta=3.31(\mathrm{t}, 4 \mathrm{H}, \mathrm{J}=7.50 \mathrm{~Hz})\right.$, $3.24(\mathrm{t}, 4 \mathrm{H}, \mathrm{J}=7.50 \mathrm{~Hz}), 2.98(\mathrm{t}, 4 \mathrm{H}, \mathrm{J}=7.03 \mathrm{~Hz}), 2.75(\mathrm{t}, 4 \mathrm{H}, \mathrm{J}=7.03 \mathrm{~Hz}), 1.49-1.59(\mathrm{~m}$, $8 \mathrm{H}), 1.24-1.31(\mathrm{~m}, 72 \mathrm{H}), 0.88-0.91(\mathrm{~m}, 12 \mathrm{H}) .{ }^{13} \mathrm{C} \mathrm{NMR}\left(126 \mathrm{MHz}, \mathrm{CDCl}_{3}\right) \delta=170.3,48.0$, 46.1, 33.6, 32.9, 31.9, 29.6, 29.6, 29.6, 29.4, 29.4, 29.4, 29.2, 27.8, 27.1, 26.9, 22.7, 14.1. HRMS (ESI): $\mathrm{m} / \mathrm{z}$ calcd for $\mathrm{C}_{54} \mathrm{H}_{108} \mathrm{~N}_{2} \mathrm{O}_{2} \mathrm{~S}_{2}\left[\mathrm{M}+\mathrm{H}^{+}\right]^{+}:$881.7925, found: 881.7939 .

\section{Branched Amides, Series II}

3,3'-disulfanediylbis(N-undecyl-N-methylamine) was synthesized according to a known protocol $^{12}$, by the methylation of undecylamine with methyl iodide. Undecylamine ( 2 mmol, 0.44 $\mathrm{mL}$ ) was dissolved in $5 \mathrm{~mL}$ of tetrahydrofuran. Triethylamine $(2 \mathrm{mmol}, 0.28 \mathrm{~mL})$ was added to 
the solution, followed by a slow, dropwise addition of methyl iodide ( $2 \mathrm{mmol}, 0.12 \mathrm{~mL})$. Water $(5 \mathrm{~mL})$ was added to the reaction, and the product was extracted from ethyl acetate $(2 \times 5 \mathrm{~mL})$. The organic layer was dried over $\mathrm{MgSO}_{4}$ and concentrated under reduced pressure to afford a white solid. The product was purified by silica gel column chromatography using a dichloromethane:methanol mixture (95:5; v:v), ${ }^{1} \mathrm{H}$ NMR $\left(500 \mathrm{MHz}, \mathrm{CDCl}_{3}\right) \delta=3.05-2.96(\mathrm{~m}$, 2H), $2.68(\mathrm{~s}, 3 \mathrm{H}), 1.92(\mathrm{ddd}, J=11.8,10.2,6.7 \mathrm{~Hz}, 2 \mathrm{H}), 1.41(\mathrm{p}, J=7.4,6.9 \mathrm{~Hz}, 2 \mathrm{H}), 1.26(\mathrm{~s}$, $14 \mathrm{H}), 0.91-0.85(\mathrm{~m}, 3 \mathrm{H})$.

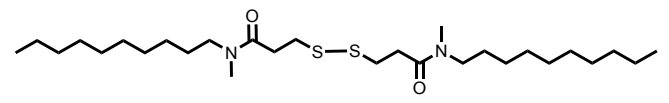

\section{3,3'-disulfanediylbis(N-undecyl-N-methylpropanamide)}

The product was purified by silica gel column chromatography with a mixture of hexanes:ethyl acetate (4:1; v:v) to afford a clear oil. ${ }^{1} \mathrm{H}-\mathrm{NMR}\left(500 \mathrm{MHz}, \mathrm{CDCl}_{3}\right): \delta=3.35(\mathrm{t}, 4 \mathrm{H}, \mathrm{J}=7.15 \mathrm{~Hz})$, $3.27(\mathrm{t}, 4 \mathrm{H}, \mathrm{J}=7.15 \mathrm{~Hz}), 2.92-2.99(\mathrm{~m}, 10 \mathrm{H}), 2.76-2.72(\mathrm{~m}, 4 \mathrm{H}), 1.46-1.59(\mathrm{~m}, 4 \mathrm{H}), 1.23-$ $1.30(\mathrm{~m}, 32 \mathrm{H}), 0.87(\mathrm{t}, 6 \mathrm{H}, \mathrm{J}=7.18 \mathrm{~Hz}) .{ }^{13} \mathrm{C} \mathrm{NMR}\left(126 \mathrm{MHz}, \mathrm{CDCl}_{3}\right) \delta=170.3,48.0,46.1$, $33.6,32.9,31.9,29.6,29.6,29.6,29.4,29.4,29.4,29.2,27.8,27.1,26.9,22.7,14.1$. HRMS (ESI): $\mathrm{m} / \mathrm{z}$ calcd for $\mathrm{C}_{54} \mathrm{H}_{108} \mathrm{~N}_{2} \mathrm{O}_{2} \mathrm{~S}_{2}\left[\mathrm{M}+\mathrm{H}^{+}\right]^{+}: 545.4169$, found: 545.4158 .

N-decyl-N-ethylpropanamide.

This intermediate not purified prior to reduction.

N-decyl-N-ethylpropanamine.

The product was purified by silica gel column chromatography with a mixture of hexanes:ethyl acetate $(4: 1 ; \mathrm{v}: \mathrm{v})$ to afford a clear oil. ${ }^{1} \mathrm{H}$ NMR $\left(500 \mathrm{MHz}, \mathrm{CDCl}_{3}\right) \delta=2.71-2.58(\mathrm{~m}, 2 \mathrm{H}), 2.54$ $-2.42(\mathrm{~m}, 2 \mathrm{H}), 1.49(\mathrm{dt}, J=16.7,8.3 \mathrm{~Hz}, 2 \mathrm{H}), 1.36-1.23(\mathrm{~m}, 14 \mathrm{H}), 1.16-0.99(\mathrm{~m}, 3 \mathrm{H}), 0.89$ 
(t, $J=6.9 \mathrm{~Hz}, 3 \mathrm{H}) .{ }^{13} \mathrm{C} \mathrm{NMR}\left(500 \mathrm{MHz}, \mathrm{CDCl}_{3}\right): \delta=49.7,44.3,31.9,30.4,29.6,29.6,29.3$,

$29.3,27.0,22.7,15.5,14.1$.

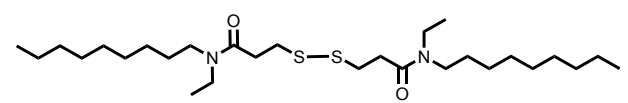

3,3'-disulfanediylbis(N-decyl-N-ethylpropanamide)

The product was purified by silica gel column chromatography with a mixture of hexanes:ethyl acetate $(4: 1 ; \mathrm{v}: \mathrm{v})$ to afford a clear oil. ${ }^{1} \mathrm{H}-\mathrm{NMR}\left(500 \mathrm{MHz}, \mathrm{CDCl}_{3}\right): \delta=3.22-3.48(\mathrm{~m}, 4 \mathrm{H}), 3.23$ $(\mathrm{t}, 4 \mathrm{H}, \mathrm{J}=7.75 \mathrm{~Hz}), 2.97(\mathrm{t}, 4 \mathrm{H}, \mathrm{J}=7.13 \mathrm{~Hz}), 2.72-2.77(\mathrm{~m}, 4 \mathrm{H}), 1.51-1.57(\mathrm{~m}, 4 \mathrm{H}), 1.25-$ $1.30(\mathrm{~m}, 28 \mathrm{H}), 1.19(\mathrm{t}, 3 \mathrm{H}, \mathrm{J}=7.04 \mathrm{~Hz}), 1.11(\mathrm{t}, 3 \mathrm{H}, \mathrm{J}=7.04 \mathrm{~Hz}), 0.86-0.89(\mathrm{~m}, 6 \mathrm{H}) .{ }^{13} \mathrm{C}-$ NMR $\left(126 \mathrm{MHz}, \mathrm{CDCl}_{3}\right): \delta=170.2,47.6,45.7,42.3,40.8,33.7,33.5,32.8,32.7,31.9,29.6$, 29.5, 29.5, 29.4, 29.4, 29.3, 29.2, 27.9, 27.1, 26.9. HRMS (ESI): m/z calcd for $\mathrm{C}_{15} \mathrm{H}_{31} \mathrm{NOS}$ $\left[\mathrm{M}+\mathrm{H}^{+}\right]^{+}:$545.4169, found: 545.4166.

N-octyl-N-butylamide.

This intermediate not purified prior to reduction.

N-octyl-N-butylamine.

The product was purified by silica gel column chromatography with a mixture of hexanes:ethyl acetate $(4: 1 ; \mathrm{v}: \mathrm{v})$ to afford a clear oil. ${ }^{1} \mathrm{H}$ NMR $\left(500 \mathrm{MHz}, \mathrm{CDCl}_{3}\right) \delta=2.64-2.56(\mathrm{~m}, 4 \mathrm{H}), 1.48$ (ddt, J = 11.7, 7.4, 4.3 Hz, 4H), $1.41-1.25(\mathrm{~m}, 12 \mathrm{H}), 0.91(\mathrm{dt}, \mathrm{J}=18.5,7.2 \mathrm{~Hz}, 6 \mathrm{H}) .{ }^{13} \mathrm{C}$ NMR $\left(500 \mathrm{MHz} \mathrm{CDCl}_{3}\right): \delta=49.9,49.6,32.7,31.9,30.4,29.3,29.3,27.0,22.7,22.1,14.1,13.8$.

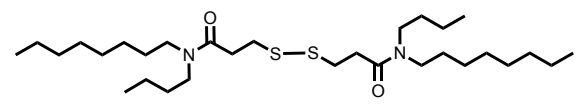

3,3'-disulfanediylbis(N-octyl-N-butylpropanamide) 
The product was purified by silica gel column with a mixture of hexanes:ethyl acetate $(4: 1 ; \mathrm{v}: \mathrm{v})$ to afford a clear oil. ${ }^{1} \mathrm{H}-\mathrm{NMR}\left(500 \mathrm{MHz}, \mathrm{CDCl}_{3}\right): \delta=3.29-3.34(\mathrm{~m}, 4 \mathrm{H}), 3.22-3.26(\mathrm{~m}, 4 \mathrm{H})$, $2.98(\mathrm{t}, 4 \mathrm{H}, \mathrm{J}=7.19 \mathrm{~Hz}), 2.75(\mathrm{t}, 4 \mathrm{H}, \mathrm{J}=7.19 \mathrm{~Hz}), 1.48-1.59(\mathrm{~m}, 8 \mathrm{H}), 1.29-1.36(\mathrm{~m}, 24 \mathrm{H})$ $0.87-0.99(\mathrm{~m}, 12 \mathrm{H}) .{ }^{13} \mathrm{C}-\mathrm{NMR}\left(126 \mathrm{MHz}, \mathrm{CDCl}_{3}\right): \delta=170.4,170.3,48.0,47.7,46.2,45.9$, 33.6, 33.6, 32.8, 31.8, 31.8, 31.2, 29.9, 29.4, 29.3, 29.5, 29.2, 29.1, 27.8, 27.1, 26.9, 26.9, 22.6, 20.3, 20.1, 14.1, 13.9, 13.8. HRMS (ESI): $\mathrm{m} / \mathrm{z}$ calcd for $\mathrm{C}_{27} \mathrm{H}_{41} \mathrm{~N}_{4}\left[\mathrm{M}+\mathrm{H}^{+}\right]^{+}: \mathrm{C}_{15} \mathrm{H}_{31} \mathrm{NOS}$ $\left[\mathrm{M}+\mathrm{H}^{+}\right]^{+}: 545.4169$, found: 545.4163 .

\section{Branched Amides, Series III}

N-dodecylacetamide.

colorless oil; 88\% yield. ${ }^{1} \mathrm{H}-\mathrm{NMR}\left(500 \mathrm{MHz}, \mathrm{CDCl}_{3}\right.$ ): $\delta 5.53$ (brs, $\left.1 \mathrm{H}, \mathrm{NH}\right), 3.24$ (q, $J=6.5 \mathrm{~Hz}$, $2 \mathrm{H}), 1.98$ (s, $3 \mathrm{H}), 1.50$ (quint, $J=6.5 \mathrm{~Hz}, 2 \mathrm{H}), 1.35-1.22(\mathrm{~m}, 18 \mathrm{H}), 0.89$ (t, $J=7.0 \mathrm{~Hz}, 3 \mathrm{H}$ ). ${ }^{13} \mathrm{C}$ NMR (500 MHz, $\left.\mathrm{CDCl}_{3}\right): \delta 170.0,39.7,31.9,29.7,29.6,29.6,29.6,29.5,29.3,29.3,26.9$, 23.3, 22.7, 14.1.

N-ethyldodecan-1-amine.

white solid/oil; 55\% yield. ${ }^{1} \mathrm{H}-\mathrm{NMR}\left(500 \mathrm{MHz}, \mathrm{CDCl}_{3}\right.$ ): 2.64 (q, $J=7.0 \mathrm{~Hz}, 2 \mathrm{H}$ ), 2.59 (t, $J=7.5$ $\mathrm{Hz}, 2 \mathrm{H}), 1.48$ (quin, $J=7.5 \mathrm{~Hz}, 2 \mathrm{H}), 1.33-1.21(\mathrm{~m}, 18 \mathrm{H}), 1.10(\mathrm{t}, J=7.0 \mathrm{~Hz}, 3 \mathrm{H}), 0.87$ (t, $J=$ $6.5 \mathrm{~Hz}, 3 \mathrm{H}) .{ }^{13} \mathrm{C} \mathrm{NMR}\left(500 \mathrm{MHz}, \mathrm{CDCl}_{3}\right): \delta=50.0,44.2,31.9,30.2,29.7,29.6,29.6,29.6$ $29.5,29.3,27.3,22.6,15.3,14.1$.

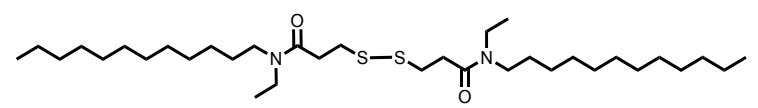

3,3'-disulfanediylbis(N-dodecyl-N-ethylpropanamide) 
The product was purified by silica gel column chromatography with a mixture of hexanes:ethyl acetate $(4: 1 ; \mathrm{v}: \mathrm{v})$ to afford a clear oil. ${ }^{1} \mathrm{H}-\mathrm{NMR}\left(500 \mathrm{MHz}, \mathrm{CDCl}_{3}\right): \delta=3.29(\mathrm{t}, 4 \mathrm{H}, \mathrm{J}=7.62 \mathrm{~Hz})$, $3.22(\mathrm{t}, 4 \mathrm{H}, \mathrm{J}=7.76 \mathrm{~Hz}), 2.96(\mathrm{t}, 4 \mathrm{H}, \mathrm{J}=7.17 \mathrm{~Hz}), 2.72(\mathrm{t}, 4 \mathrm{H}, \mathrm{J}=7.17 \mathrm{~Hz}), 1.47-1.59(\mathrm{~m}$, $8 \mathrm{H}), 1.26-1.30(\mathrm{~m}, 32 \mathrm{H}), 0.87-0.98(\mathrm{~m}, 12 \mathrm{H}) .{ }^{13} \mathrm{C}-\mathrm{NMR}\left(126 \mathrm{MHz}, \mathrm{CDCl}_{3}\right): \delta=170.2,47.6$, $45.7,42.3,40.8,33.7,33.5,32.8,32.7,31.9,29.6,29.5,29.5,29.4,29.4,29.3,29.2,27.9,27.1$, 26.9. 22.6, 14.1. HRMS (ESI): $\mathrm{m} / \mathrm{z}$ calcd for $\mathrm{C}_{34} \mathrm{H}_{68} \mathrm{~N}_{2} \mathrm{O}_{2} \mathrm{~S}_{2}\left[\mathrm{M}+\mathrm{H}^{+}\right]^{+}: 601.4795$, found: 601.4800.

N-dodecylbutyramide.

white solid; 90\% yield. ${ }^{1} \mathrm{H}-\mathrm{NMR}\left(500 \mathrm{MHz}, \mathrm{CDCl}_{3}\right.$ ): $\delta 5.52$ (brs, $1 \mathrm{H}, \mathrm{NH}$ ), 3.25 (q, $J=7.0 \mathrm{~Hz}$, $J=6.5 \mathrm{~Hz}, 2 \mathrm{H}$ ), $2.15(\mathrm{t}, J=7.5 \mathrm{~Hz}, 2 \mathrm{H}), 1.67$ (sex, $J=7.5 \mathrm{~Hz}, 2 \mathrm{H}$ ), 1.50 (quint, $J=7.0 \mathrm{~Hz}, J=$ $6.5 \mathrm{~Hz}, 2 \mathrm{H}), 1.35-1.22(\mathrm{~m}, 18 \mathrm{H}), 0.96(\mathrm{t}, J=7.5 \mathrm{~Hz}, 3 \mathrm{H}), 0.89(\mathrm{t}, J=7.5 \mathrm{~Hz}, 3 \mathrm{H}) .{ }^{13} \mathrm{C}$ NMR (500 MHz, $\left.\mathrm{CDCl}_{3}\right): \delta=172.9,39.5,38.8,31.9,29.7,29.6,29.6,29.6,29.5,29.3,29.3,26.9,22.7$, 19.2, 14.1, 13.8.

N-butyldodecan-1-amine.

white solid/oil; 9.4\% yield. ${ }^{1} \mathrm{H}-\mathrm{NMR}$ (500 MHz, $\mathrm{CDCl}_{3}$ ): $\delta$ 2.62-2.55 (m, $4 \mathrm{H}$ ), 1.53-1.44 (m, 4 H), 1.36-1.15 (m, $20 \mathrm{H}), 0.88$ (t, $J=7.5 \mathrm{~Hz}, 3 \mathrm{H}), 0.84$ (t, $J=7.0 \mathrm{~Hz}, 3 \mathrm{H}) .{ }^{13} \mathrm{C}$ NMR $(500 \mathrm{MHz}$, $\left.\mathrm{CDCl}_{3}\right): \delta=49.8,49.4,31.9,31.7,29.6,29.6,29.6,29.5,29.3,27.3,22.6,20.4,14.0,13.9$.

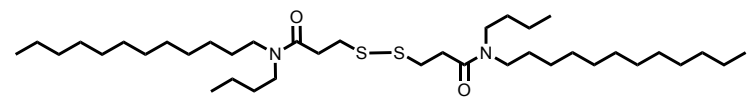

3,3'-disulfanediylbis(N-dodecyl-N-butylpropanamide)

The product was purified by silica gel column chromatography with a mixture of hexanes:ethyl acetate $(4: 1 ; \mathrm{v}: \mathrm{v})$ to afford a clear oil. ${ }^{1} \mathrm{H}-\mathrm{NMR}\left(500 \mathrm{MHz}, \mathrm{CDCl}_{3}\right): \delta=3.31(\mathrm{t}, 4 \mathrm{H}, \mathrm{J}=7.96 \mathrm{~Hz})$, $3.24(\mathrm{t}, 4 \mathrm{H}, \mathrm{J}=7.96 \mathrm{~Hz}), 2.98(\mathrm{t}, 4 \mathrm{H}, \mathrm{J}=7.03 \mathrm{~Hz}), 2.75(\mathrm{t}, 4 \mathrm{H}, \mathrm{J}=7.03 \mathrm{~Hz}), 1.48-1.59(\mathrm{~m}$, 
$8 \mathrm{H}), 1.26-1.34(\mathrm{~m}, 40 \mathrm{H}), 0.86-0.91(\mathrm{~m}, 12 \mathrm{H}) .{ }^{13} \mathrm{C}-\mathrm{NMR}\left(126 \mathrm{MHz}, \mathrm{CDCl}_{3}\right): \delta=170.4$, $170.3,48.0,47.7,46.2,45.9,33.6,33.6,32.8,31.8,31.8,31.2,29.9,29.4,29.3,29.5,29.2,29.1$, 27.8, 27.1, 26.9, 26.9, 22.6, 20.3, 20.1, 14.1, 13.9, 13.8. HRMS (ESI): $\mathrm{m} / \mathrm{z}$ calcd for $\mathrm{C}_{38} \mathrm{H}_{76} \mathrm{~N}_{2} \mathrm{O}_{2} \mathrm{~S}_{2}\left[\mathrm{M}+\mathrm{H}^{+}\right]^{+}:$657.5421, found: 657.5415 .

N-dodecylhexanamide.

white solid; $51.6 \%$ yield. ${ }^{1} \mathrm{H}-\mathrm{NMR}\left(500 \mathrm{MHz}, \mathrm{CDCl}_{3}\right.$ ): $\delta 5.44$ (brs, $\left.1 \mathrm{H}, \mathrm{NH}\right), 3.25$ (q, $J=7.0 \mathrm{~Hz}$, $2 \mathrm{H}$ ), 2.16 (t, $J=7.5 \mathrm{~Hz}, 2 \mathrm{H}$ ), 1.64 (quint, $J=7.5 \mathrm{~Hz}, 2 \mathrm{H}$ ), 1.50 (quint, $J=7.0 \mathrm{~Hz}, 2 \mathrm{H}$ ), 1.37 $1.22(\mathrm{~m}, 22 \mathrm{H}), 0.93-0.87(\mathrm{~m}, 6 \mathrm{H}) .{ }^{13} \mathrm{C}$ NMR (500 MHz, $\left.\mathrm{CDCl}_{3}\right): \delta 173.0,39.5,36.9,31.9,31.5$, 29.7, 29.6, 29.6, 29.6, 29.5, 29.3, 29.3, 26.9, 25.3, 22.7, 22.4, 14.1, 14.0.

N-hexyldodecan-1-amine.

white solid/oil; $16.3 \%$ yield. ${ }^{1} \mathrm{H}-\mathrm{NMR}\left(500 \mathrm{MHz}, \mathrm{CDCl}_{3}\right): \delta$ 2.61-2.55 (m, $\left.4 \mathrm{H}\right), 1.51-1.44(\mathrm{~m}, 4$ H), 1.35-1.21 (m, $24 \mathrm{H}), 0.92-0.85$ (m, $6 \mathrm{H}) .{ }^{13} \mathrm{C}$ NMR (500 MHz, $\left.\mathrm{CDCl}_{3}\right): \delta=50.2,50.1,31.9$, $30.2,30.1,29.7,29.6,29.6,29.6,29.5,29.3,27.4,27.1,22.7,22.6,14.1,14.0$.

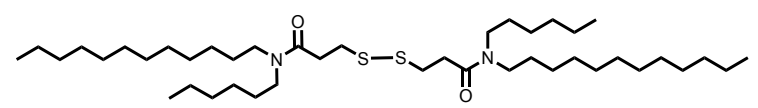

3,3'-disulfanediylbis(N-dodecyl-N-hexylpropanamide)

The product was purified by silica gel column chromatography with a mixture of hexanes:ethyl acetate $(4: 1 ; \mathrm{v}: \mathrm{v})$ to afford a clear oil. ${ }^{1} \mathrm{H}-\mathrm{NMR}\left(500 \mathrm{MHz}, \mathrm{CDCl}_{3}\right): \delta=3.31(\mathrm{t}, 4 \mathrm{H}, \mathrm{J}=7.96 \mathrm{~Hz})$, $3.24(\mathrm{t}, 4 \mathrm{H}, \mathrm{J}=7.96 \mathrm{~Hz}), 2.98(\mathrm{t}, 4 \mathrm{H}, \mathrm{J}=7.03 \mathrm{~Hz}), 2.75(\mathrm{t}, 4 \mathrm{H}, \mathrm{J}=7.03 \mathrm{~Hz}), 1.48-1.59(\mathrm{~m}$, $8 \mathrm{H}), 1.26-1.34(\mathrm{~m}, 48 \mathrm{H}), 0.86-0.91(\mathrm{~m}, 12 \mathrm{H}) .{ }^{13} \mathrm{C}-\mathrm{NMR}\left(126 \mathrm{MHz}, \mathrm{CDCl}_{3}\right): \delta=170.4,170.3$, 48.0, 47.7, 46.2, 45.9, 33.6, 33.6, 32.8, 31.8, 31.8, 31.2, 29.9, 29.4, 29.3, 29.5, 29.2, 29.1, 27.8, 27.1, 26.9, 26.9, 22.6, 20.3, 20.1, 14.1, 13.8. HRMS (ESI): $\mathrm{m} / \mathrm{z}$ calcd for $\mathrm{C}_{42} \mathrm{H}_{54} \mathrm{~N}_{2} \mathrm{O}_{2} \mathrm{~S}_{2}$ $\left[\mathrm{M}+\mathrm{H}^{+}\right]^{+}:$713.6047, found: 713.6054 . 
N-dodecyloctanamide.

white solid; 10.4\% yield. ${ }^{1} \mathrm{H}-\mathrm{NMR}\left(500 \mathrm{MHz}, \mathrm{CDCl}_{3}\right): \delta 5.41$ (brs, $\left.1 \mathrm{H}, \mathrm{NH}\right), 3.15$ (q, J=7.0 Hz, $2 \mathrm{H}), 2.07$ (t, $J=7.5 \mathrm{~Hz}, 2 \mathrm{H}$ ), 1.54 (quint, $J=7.5 \mathrm{~Hz}, 2 \mathrm{H}$ ), 1.40 (quint, $J=7.0 \mathrm{~Hz}, 2 \mathrm{H}$ ), 1.27 -

$1.13(\mathrm{~m}, 26 \mathrm{H}), 0.82-0.77(\mathrm{~m}, 6 \mathrm{H}) .{ }^{13} \mathrm{C} \mathrm{NMR}\left(500 \mathrm{MHz}, \mathrm{CDCl}_{3}\right): \delta 173.0,39.4,36.8,31.8,31.6$, $29.6,29.6,29.5,29.5,29.5,29.3,29.2,29.2,28.9,26.8,25.8,22.6,22.5,14.0,14.0$.

N-octyldodecan-1-amine.

white solid/oil; 80.9\% yield. ${ }^{1} \mathrm{H}-\mathrm{NMR}\left(500 \mathrm{MHz}, \mathrm{CDCl}_{3}\right): \delta 2.62-2.57(\mathrm{~m}, 4 \mathrm{H}), 1.54-1.45$ (m, 4 $\mathrm{H}), 1.37-1.21(\mathrm{~m}, 28 \mathrm{H}), 0.93-0.86(\mathrm{~m}, 6 \mathrm{H}) .{ }^{13} \mathrm{C} \mathrm{NMR}\left(500 \mathrm{MHz}, \mathrm{CDCl}_{3}\right): \delta=50.1,50.1,31.9$ $31.8,30.2,30.1,29.7,29.6,29.6,29.6,29.5,29.4,29.3,29.2,27.4,27.1,22.7,22.7,14.1,14.1$.

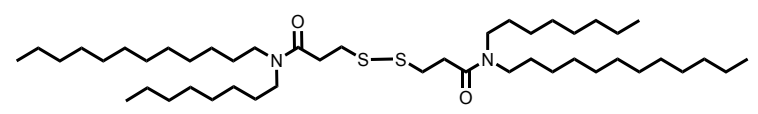

3,3'-disulfanediylbis(N-dodecyl-N-octylpropanamide)

The product was purified by silica gel column chromatography with a mixture of hexanes:ethyl acetate (4:1; v:v) to afford a clear oil. ${ }^{1} \mathrm{H}-\mathrm{NMR}\left(500 \mathrm{MHz}, \mathrm{CDCl}_{3}\right): \delta=3.31(\mathrm{t}, 4 \mathrm{H}, \mathrm{J}=7.74 \mathrm{~Hz})$, $3.24(\mathrm{t}, 4 \mathrm{H}, \mathrm{J}=7.74 \mathrm{~Hz}), 2.98(\mathrm{t}, 4 \mathrm{H}, \mathrm{J}=7.19 \mathrm{~Hz}), 2.75(\mathrm{t}, 4 \mathrm{H}, \mathrm{J}=7.19 \mathrm{~Hz}), 1.49-1.60(\mathrm{~m}$, $8 \mathrm{H}), 1.26-1.31(\mathrm{~m}, 56 \mathrm{H}), 0.84-0.92(\mathrm{~m}, 12 \mathrm{H}) .{ }^{13} \mathrm{C}-\mathrm{NMR}\left(126 \mathrm{MHz}, \mathrm{CDCl}_{3}\right): \delta=170.4,170.3$, 48.0, 47.7, 46.2, 45.9, 33.6, 33.6, 32.8, 31.8, 31.8, 31.2, 29.9, 29.4, 29.3, 29.5, 29.2, 29.1, 27.8, 27.1, 26.9, 26.9, 22.6, 20.3, 20.1, 14.1, 13.9. HRMS (ESI): $\mathrm{m} / \mathrm{z}$ calcd for $\mathrm{C}_{46} \mathrm{H}_{92} \mathrm{~N}_{2} \mathrm{O}_{2} \mathrm{~S}_{2}$ $\left[\mathrm{M}+\mathrm{H}^{+}\right]^{+}:$769.6673, found: 769.6671.

N-dodecyldecanamide. 
white solid; $8.5 \%$ yield. ${ }^{1} \mathrm{H}-\mathrm{NMR}$ (500 MHz, $\mathrm{CDCl}_{3}$ ): $\delta 5.45$ (brs, $1 \mathrm{H}, \mathrm{NH}$ ), 3.25 (q, $J=6.5 \mathrm{~Hz}$, $2 \mathrm{H}$ ), 2.17 (t, $J=7.5 \mathrm{~Hz}, 2 \mathrm{H}$ ), 1.63 (quint, $J=7.5 \mathrm{~Hz}, 2 \mathrm{H}$ ), 1.50 (quint, $J=6.5 \mathrm{~Hz}, 2 \mathrm{H}$ ), 1.37 $1.22(\mathrm{~m}, 30 \mathrm{H}), 0.93-0.87(\mathrm{~m}, 6 \mathrm{H}) .{ }^{13} \mathrm{C} \mathrm{NMR}\left(500 \mathrm{MHz}, \mathrm{CDCl}_{3}\right): \delta 173.2,39.5,36.9,31.9,31.9$, 29.7, 29.7, 29.6, 29.6, 29.5, 29.4, 29.4, 29.4, 29.3, 29.3, 29.2, 26.9, 25.9, 22.7, 22.7, 14.1, 14.1 . N-decyldodecan-1-amine.

white solid/oil; 70\% yield. ${ }^{1} \mathrm{H}-\mathrm{NMR}\left(500 \mathrm{MHz}, \mathrm{CDCl}_{3}\right): \delta 2.60-2.54(\mathrm{~m}, 4 \mathrm{H}), 1.51-1.43$ (m, 4 $\mathrm{H}), 1.34-1.15(\mathrm{~m}, 32 \mathrm{H}), 0.89-0.83(\mathrm{~m}, 6 \mathrm{H}) .{ }^{13} \mathrm{C} \mathrm{NMR}\left(500 \mathrm{MHz}, \mathrm{CDCl}_{3}\right): \delta=50.1,50.1,31.9$, $31.8,30.2,30.1,29.7,29.7,29.6,29.6,29.6,29.6,29.5,29.4,29.3,29.2,27.4,27.1,22.7,22.7$, 14.1, 14.1.

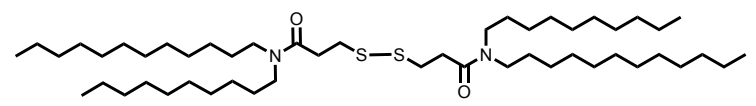

3,3'-disulfanediylbis(N-dodecyl-N-decylpropanamide)

The product was purified by silica gel column chromatography with a mixture of hexanes:ethyl acetate $(4: 1 ; \mathrm{v}: \mathrm{v})$ to afford a clear oil. ${ }^{1} \mathrm{H}-\mathrm{NMR}\left(500 \mathrm{MHz}, \mathrm{CDCl}_{3}\right): \delta=3.30(\mathrm{t}, 4 \mathrm{H}, \mathrm{J}=7.54 \mathrm{~Hz})$, $3.24(\mathrm{t}, 4 \mathrm{H}, \mathrm{J}=7.54 \mathrm{~Hz}), 2.98(\mathrm{t}, 4 \mathrm{H}, \mathrm{J}=7.23 \mathrm{~Hz}), 2.75(\mathrm{t}, 4 \mathrm{H}, \mathrm{J}=7.23 \mathrm{~Hz}), 1.47-1.59(\mathrm{~m}$, $8 \mathrm{H}), 1.26-1.30(\mathrm{~m}, 64 \mathrm{H}), 0.87-0.92(\mathrm{~m}, 12 \mathrm{H}) .{ }^{13} \mathrm{C}-\mathrm{NMR}\left(126 \mathrm{MHz}, \mathrm{CDCl}_{3}\right): \delta=170.4,170.3$, 48.0, 47.7, 46.2, 45.9, 33.6, 33.6, 32.8, 31.8, 31.8, 31.2, 29.9, 29.4, 29.3, 29.5, 29.2, 29.1, 27.8, 27.1, 26.9, 26.9, 22.6, 20.3, 20.1, 14.1, 14.0. HRMS (ESI): $\mathrm{m} / \mathrm{z}$ calcd for $\mathrm{C}_{50} \mathrm{H}_{100} \mathrm{~N}_{2} \mathrm{O}_{2} \mathrm{~S}_{2}$ $\left[\mathrm{M}+\mathrm{H}^{+}\right]^{+}: 825.7299$ found: 825.7295 . 


\section{S12. Contact angle data}

Table S15. Measured contact angles for a selected set of SAMs. The measurements were performed on freshly prepared SAMs with a Ramé-Hart goniometer using $<10 \mu \mathrm{L}$ of deionized water (Milli-Q, resistivity $<18 \mathrm{M} \Omega \cdot \mathrm{cm})$. Advancing and receding contact angles are reported as the mean and standard deviation of a total of 12 measurements per sample.

\begin{tabular}{ccccc} 
SAMPLE & $\begin{array}{c}\text { Advancing } \\
\text { Contact Angle }\left(^{\circ}\right)\end{array}$ & $\begin{array}{c}\text { Standard } \\
\text { Deviation }\end{array}$ & $\begin{array}{c}\text { Receding Contact } \\
\text { Angle }\left(^{\circ}\right)\end{array}$ & $\begin{array}{c}\text { Standard } \\
\text { Deviation }\end{array}$ \\
\hline $\mathbf{5}_{(12, \mathrm{H})}$ & 99 & 2.8 & 99 & 3.18 \\
$\mathbf{7}_{(6,6)}$ & 85.5 & 0.37 & 84.3 & 0.09 \\
$\mathbf{1 0}_{(12,12)}$ & 101 & 4.3 & 99 & 2.1 \\
$\mathbf{1 1}_{(8,4)}$ & 93 & 1.9 & 92 & 3.07 \\
$\mathbf{1 2}_{(10,2)}$ & 98.4 & 0.6 & 95.9 & 0.44 \\
$\mathbf{1 3}_{(11,1)}$ & 105.4 & 0.24 & 107 & 1.3 \\
$\mathbf{1 4}_{(12,2)}$ & 94 & 1.68 & 92 & 3.4 \\
$\mathbf{1 6}_{(12,6)}$ & 82 & 2.11 & 84 & 3.99 \\
$\mathbf{1 8}_{(12,10)}$ & 100.9 & 0.61 & 99.3 & 0.2
\end{tabular}




\section{References}

1. Barber, J. R., Yoon, Hyo Jae, Bowers, Carleen M., Thuo, Martin M., Breiten, Benjamin, Gooding, Diana M., Whitesides, George M., The influence of environmental factors on the measurement of rates of charge transport across $\mathrm{Ag}^{\mathrm{TS}} / \mathrm{SAM} / / \mathrm{Ga}_{2} \mathrm{O}_{3} / \mathrm{EGaIn}$ junctions. Chem. Mater. 2014, 26, 3938-3947.

2. $\quad$ Simeone, F. C.; Yoon, H. J.; Thuo, M. M.; Barber, J. R.; Smith, B.; Whitesides, G. M., Defining the value of injection current and effective electrical contact area for egain-based molecular tunneling junctions. J. Am. Chem. Soc. 2013, 135, 18131-18144.

3. Reus, W. F.; Thuo, M. M.; Shapiro, N. D.; Nijhuis, C. A.; Whitesides, G. M., The SAM, Not the electrodes, dominates charge transport in metal-monolayer/Ga2O3/Gallium-Indium eutectic junctions. ACS Nano 2012, 6, 4806-4822.

4. Ghorai, P. K.; Glotzer, S. C. Molecular dynamics simulation study of self-assembled monolayers of alkanethiol surfactants on spherical gold nanoparticles. J. Phys. Chem. C 2007, $111,15857-15862$.

5. Weiss, E. A.; Kaufman, G. K.; Kriebel, J. K.; Li, Z.; Schalek, R.; Whitesides, G. M., $\mathrm{Si} / \mathrm{SiO} 2$-templated formation of ultraflat metal surfaces on glass, polymer, and solder supports: their use as substrates for self-assembled monolayers. Langmuir 2007, 23, 9686-9694.

6. Thuo, M. M.; Reus, W. F.; Simeone, F. C.; Kim, C.; Schulz, M. D.; Yoon, H. J.; Whitesides, G. M., Replacing-CH2CH2- with -CONH- does not significantly change rates of charge transport through Ag-TS-SAM//Ga2O3/EGaIn junctions. J. Am. Chem. Soc. 2012, 134, 10876-10884.

7. Love, J. C.; Estroff, L. A.; Kriebel, J. K.; Nuzzo, R. G.; Whitesides, G. M., Selfassembled monolayers of thiolates on metals as a form of nanotechnology. Chem. Rev. 2005, 105, 1103-1169.

8. $\quad$ Freeman, T. L.; Evans, S. D.; Ulman, A., XPS studies of self-assembled multilayer films. Langmuir 1995, 11, 4411-4417.

9. Zhang, Q.; Huang, H.; He, H.; Chen, H.; Shao, H.; Liu, Z., Determination of locations of sulfur, amide-nitrogen and azo-nitrogen in self-assembled monolayers of alkanethiols and azobenzenethiols on Au (111) and GaAs (100) by angle-resolved X-ray photoelectron spectroscopy. Surf. Sci. 1999, 440, 142-150.

10. Schlenoff, J. B.; Li, M.; Ly, H., Stability and self-exchange in alkanethiol monolayers. $J$. Am. Chem. Soc. 1995, 117 (50), 12528-12536.

11. Yan, C.; Yuan, R.; Pfalzgraff, W. C.; Nishida, J.; Wang, L.; Markland, T. E.; Fayer, M. D., Unraveling the dynamics and structure of functionalized self-assembled monolayers on gold using 2D IR spectroscopy and MD simulations. Proc. Natl. Acad. Sci. USA 2016, 113, 49294934.

12. Milelli, A.; Marchetti, C.; Greco, M. L.; Moraca, F.; Costa, G.; Turrini, E.; Catanzaro, E.; Betari, N.; Calcabrini, C.; Sissi, C.; Alcaro, S.; Fimognari, C.; Tumiatti, V.; Minarini, A., Naphthalene diimide-polyamine hybrids as antiproliferative agents: Focus on the architecture of the polyamine chains. Eur. J. Med. Chem. 2017, 128, 107-122. 